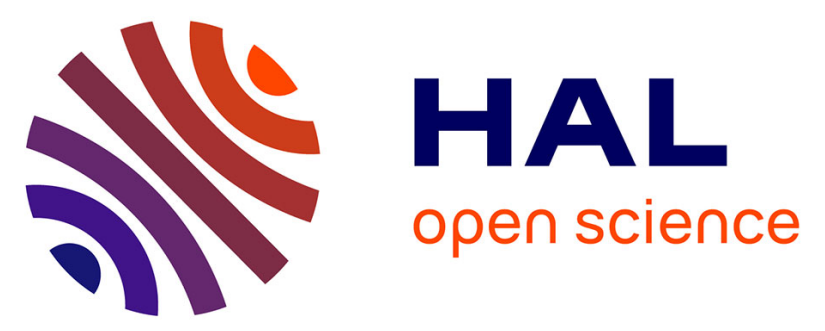

\title{
Finite element modeling of grain size effects on the ultrasonic microstructural noise backscattering in polycrystalline materials
}

\author{
Xue Bai, B. Tie, D. Aubry, Jean-Hubert Schmitt
}

\section{To cite this version:}

Xue Bai, B. Tie, D. Aubry, Jean-Hubert Schmitt. Finite element modeling of grain size effects on the ultrasonic microstructural noise backscattering in polycrystalline materials. Ultrasonics, 2018, 87, pp.182-202. 10.1016/j.ultras.2018.02.008 . hal-01832543

\section{HAL Id: hal-01832543 \\ https://hal.science/hal-01832543}

Submitted on 10 Dec 2021

HAL is a multi-disciplinary open access archive for the deposit and dissemination of scientific research documents, whether they are published or not. The documents may come from teaching and research institutions in France or abroad, or from public or private research centers.
L'archive ouverte pluridisciplinaire HAL, est destinée au dépôt et à la diffusion de documents scientifiques de niveau recherche, publiés ou non, émanant des établissements d'enseignement et de recherche français ou étrangers, des laboratoires publics ou privés.

\section{(ㄷ)(1) $\$$}

Distributed under a Creative Commons Attribution - NonCommerciall 4.0 International 


\section{Accepted Manuscript}

Finite element modeling of grain size effects on the ultrasonic microstructural noise backscattering in polycrystalline materials

X. Bai, B. Tie, J.-H. Schmitt, D. Aubry

PII:

S0041-624X(18)30106-9

DOI: https://doi.org/10.1016/j.ultras.2018.02.008

Reference:

ULTRAS 5701

To appear in: $\quad$ Ultrasonics

Received Date: $\quad 26$ August 2016

Revised Date: $\quad 6$ February 2018

Accepted Date: 7 February 2018

Please cite this article as: X. Bai, B. Tie, J.-H. Schmitt, D. Aubry, Finite element modeling of grain size effects on the ultrasonic microstructural noise backscattering in polycrystalline materials, Ultrasonics (2018), doi: https:// doi.org/10.1016/j.ultras.2018.02.008

This is a PDF file of an unedited manuscript that has been accepted for publication. As a service to our customers we are providing this early version of the manuscript. The manuscript will undergo copyediting, typesetting, and review of the resulting proof before it is published in its final form. Please note that during the production process errors may be discovered which could affect the content, and all legal disclaimers that apply to the journal pertain. 


\title{
Finite element modeling of grain size effects on the ultrasonic microstructural noise backscattering in polycrystalline materials
}

\author{
X. Bai, B. Tie ${ }^{l}$, J.-H. Schmitt, D. Aubry \\ MSSMat, CNRS, CentraleSupélec, Université Paris-Saclay, 3 Rue Joliot Curie, 91190 Gif-sur-Yvette, France
}

\begin{abstract}
The correlation between ultrasonic wave propagation and polycrystalline microstructures has significant implications in nondestructive evaluation. An original numerical approach using the finite element method to quantify in both time and frequency domains the ultrasonic noise scattering due to the elastic heterogeneity of polycrystalline microstructures is presented. Based on the reciprocity theorem, it allows the scattering evaluation using mechanical data recorded only on the boundary of polycrystal instead of within its volume and is applicable to any polycrystalline aggregate regardless of its crystallographic or morphological characteristics. Consequently it gives a more realistic and accurate access of polycrystalline microstructures than the classical analytical models developed under the assumption of single scattering and the Born approximation.

The numerical approach is proposed within the same unified theoretical framework as the classical analytical models, so it is possible to validate it in the cases of idealized microstructures for which the considered analytical models remain relevant. As an original result, assuming single phase, untextured and equiaxed microstructures, two-dimensional (2D) theoretical formulas are developed and a frequency-dependent coefficient is found compared to the classical three-dimensional (3D) formulas. 2D numerical simulations are then performed for idealized microstructures composed of hexagonal grains with a uniform grain-size. Three grain sizes are considered herein and involve different scattering regions. Good comparisons are obtained between theoretical and numerical estimates of the backscattering coefficient, which validate the numerical approach. Effects of the grain boundary orientations are analyzed by modeling an irregular hexagonal grain morphology and a random grain morphology generated by an established Voronoi approach. The origin of the significant oscillation level of backscattering is then investigated and discussed.
\end{abstract}

Keywords: Ultrasonic noise backscattering, Polycrystalline materials, Grain size effects, Reciprocity theorem, Elastic wave propagation, Finite element modeling.

\footnotetext{
${ }^{1}$ Corresponding author.

E-mail: bing.tie@centralesupelec.fr
} 


\section{Introduction}

The motivation for the research on the ultrasonic wave propagation in polycrystalline materials is found in nondestructive evaluation (NDE). When ultrasonic waves propagate through polycrystalline materials, the incident energy is scattered by grain boundaries due to heterogeneities of mechanical properties, such as the elastic stiffness tensor and the density. Two phenomena therefore occur: amplitude attenuations and microstructural noise signals. Important theoretical investigations have been done for the scattering induced attenuation (e.g. [1]-[5]). In some circumstances the NDE measurement can be restricted by uncontrolled high noise-to-signal ratio, for it is difficult or even impossible to identify echo signals of interest when their amplitudes are of the same order of magnitude as noise level. Thus efforts have been dedicated to the study of scattered noise signals in order to improve nondestructive inspection and evaluation of microstructures and of their evolution.

Theoretical developments to evaluate the scattering effectiveness are based on two seminal works:

The first one initiated by Gubernatis et al. [1] is based on a single-scattering assumption and provides the solutions of scattering amplitudes of ultrasonic waves caused by a bounded isolated scatter embedded in an infinite isotropic and homogeneous elastic medium. To obtain explicit formulas for the scattering coefficient, the Born approximation was applied and the author concluded that the theoretical formulas were valid only for frequencies below the very high frequency range, often called the geometric region [6]. Based on the same approach, further theoretical investigations were proposed by Han, predicting the ultrasonic backscattering in duplex microstructures composed of randomly-oriented macrograins composed of colonies with crystallographically related orientations [7].

The second method is based on the reciprocity theorem, initially used to prove the interchangeability between a source and a receiver in the electromagnetic wave and elastic wave transmission problems [8]. Kino adapted the scattering matrix defined for electromagnetic waves to elastic waves to derive scattering formulas and made a fundamental contribution to the application of the reciprocity principle to the scattering of elastic waves by flaws [9]. Finally, explicit formulas of backscattering coefficient were proposed by Rose for single-phase and macroscopically isotropic and homogeneous polycrystalline materials with the use of the Born approximation in three-dimensional cases [10].

The equivalence of both methods was proven by Rose [10] and Margetan et al. [11] with the assumption that the elastic properties of the macroscopic effective medium are defined by the unweighted Voigt average of the elastic properties of the polycrystalline microstructure and in the weak scattering case for which the use of the Born approximation is pertinent. Analytical formulas for backscattering in equiaxed polycrystals under the single-scattering assumption were developed [10] and extended to general pitch-catch configurations [11]. More recently, Ghoshal et al. developed a multiple-scattering framework using the Wigner distribution [12]. Based on this multiple-scattering 
framework, a model for double scattering is recently developed by Hu et al. [13], which showed a strong potential to analyze strongly scattering materials.

However, despite the theoretical results, significant difficulties still remain to develop analytical formulas of ultrasonic scattering coefficients in polycrystalline materials with a more complex microstructure. Therefore it is believed that numerical modeling should be a powerful alternative to access more real and complex polycrystalline microstructures and to improve the understanding of their interactions with ultrasonic waves. Among possible numerical methods, full-field numerical modeling using the finite element method (FEM) allows taking into account polycrystalline microstructures without any simplifying assumptions and therefore grasping of complex phenomena of wave propagation in polycrystals, especially effects of the multiple scattering [14-16]. Finite element modeling is applicable to quantify the scattering effectiveness due to grain boundaries of numerically generated polycrystalline microstructures. Furthermore, progress in the electron backscatter diffraction (EBSD) technique makes possible to obtain input maps of real microstructures to take into account by finite element meshes [17]. Its accuracy and versatility in modeling numerical or real polycrystalline microstructures make the finite element method a promising tool for further developments of nondestructive inspection.

Former works on finite element simulations focused on the evaluation of ultrasonic attenuation in polycrystalline materials, e.g. $[14,15,18,19]$. However there is relatively little work on the development of methods based on finite element full-field modeling, which allows precise evaluation of microstructural noise levels [20]. The purpose of the present work is to propose a versatile FE approach that leads to an evaluation of scattering effectiveness in an arbitrary spatial direction in any polycrystalline medium. The FE approach is developed within the unified framework based on the reciprocity theorem. Before being applied for analyzing experimental results, it must be checked the proposed modeling avoids any numerical artifacts. Therefore it is validated by comparison with the classical theoretical models in the case of analyzing grain size effects on the ultrasonic backscattering in single phase, untextured and equiaxed polycrystals with idealized but representative theoretical microstructures.

Only 2D FE simulations are conducted. To exclude the grain size distribution effects [21], especially at the transition between the different regimes as, for instance, Rayleigh-to-stochastic, microstructures with numerically generated single-size regular hexagonal grains are mainly considered. This is obviously far from the actual grain structure in a polycrystal, but it gives a kind of asymptotic result. Such a hypothesis leads obviously to the periodicity of the grain morphology, possibly causing numerical artifacts. Two other cases of microstructures with geometrically varying grains are therefore discussed: an irregular hexagonal grain morphology and a Voronoi tessellation, both of which have a narrow dispersion of grain size and are geometrically close to a naturally occurring polycrystalline microstructure [15]. An external pressure loading is used to produce an incident signal, which 
simulates, for instance, the broadband laser-generated ultrasound pulse [22]. Frequency content of the incident signal is large enough to account for different scattering regions with respect to three studied grains sizes. This example of laser-generated ultrasonic waves also justifies a first 2D numerical approach, since it is of significance to the cases where transducers with a cylindrical focus are used for the inspection of heterogeneous materials [20]. This is, for instance, the case for ultrasonic characterization of the microstructure using a laser pulse following the work by Monchalin [23]. The backscattering coefficient is calculated in both time and frequency domains using numerically recorded data at several receivers placed at the boundary of the polycrystals. Dependence of the backscattering coefficient on the frequency and the grain size are therefore analyzed and compared with theoretical predictions.

The paper is organized as follows: A unified framework for theoretical and numerical evaluations of noise scattering is presented in Section 2. Classical analytical models are recalled, 2D analytical formulas of the backscattering coefficient are developed, especially the constant of proportionality between the grain-noise scattering coefficient and the received power due to the grain scattering proposed and already identified by Margetan et al. [11] in the 3D case is derived. The ratio of this constant between the $2 \mathrm{D}$ and $3 \mathrm{D}$ cases is proved to be frequency dependent. Numerical measure procedure is then proposed. In Section 3, FE ultrasonic propagation models for backscattering measurement are defined, quality analysis of numerical simulations is presented with a mesh convergence analysis and a study of phase velocities in polycrystalline materials. Section 4 presents numerical results concerning the grain size effects on the ultrasonic backscattering coefficient, followed by some main conclusions given in Section 5 .

\section{A unified framework for theoretical and numerical evaluation}

A unified theoretical framework based on the reciprocity theorem for the evaluation of the ultrasonic noise scattering in both 2D and 3D cases is presented. Analytical formulas for the scattering coefficient are obtained by considering the equivalence between the amplitudes of scattered noise signals obtained using two different methods: the one based on the well-known Auld's electromechanical reciprocity relation, which adapts the reciprocity theorem to the evaluation of scattering coefficients using transducer output signals [8,9], and the other one based on far-field analysis of an isolated scatterer, which also refers to the reciprocity theorem for obtaining full elastodynamic wave fields [24]. The key assumption is the weak scattering so the Born approximation can be applied.

As a principal result of this work, a numerical evaluation approach is defined within the unified framework of Auld's reciprocity theorem and the corresponding expressions of the ultrasonic noise backscattering coefficient using FE modeling in both 2D and 3D cases are proposed. 


\subsection{Reciprocity gap due to the microstructural scattering}

Consider a polycrystalline material occupying a region $\Omega$ of space dimension $N_{\operatorname{dim}}(=2,3)$ and bounded by a surface $\partial \Omega$ and defined by a position-dependent elastic stiffness tensor $\boldsymbol{C}(\boldsymbol{x})$ and a position-dependent density field $\rho(\boldsymbol{x})$ (Fig. 1.). Applying the Fourier transform to the elastic wave equation, the Helmholtz equation without any source term inside $\Omega$ can be written as:

$$
\operatorname{Div}_{\boldsymbol{x}} \boldsymbol{\sigma}(\boldsymbol{x})+\rho \omega^{2} \boldsymbol{u}(\boldsymbol{x})=0
$$

where $\mathbf{D i v}_{\boldsymbol{x}}$ denotes the usual divergence operator of a second order tensor with respect to the position $\boldsymbol{x}, \boldsymbol{\sigma}(\boldsymbol{x})=\boldsymbol{C}(\boldsymbol{x}): \boldsymbol{\varepsilon}(\boldsymbol{x})$ is the stress tensor, $\boldsymbol{\varepsilon}(\boldsymbol{x})=\frac{1}{2}\left(\boldsymbol{\nabla}_{x}(\boldsymbol{u}(\boldsymbol{x}))+\boldsymbol{\nabla}_{\boldsymbol{x}}^{\mathrm{T}}(\boldsymbol{u}(\boldsymbol{x}))\right)$ is the strain tensor and $\omega$ denotes the angular frequency. Herein tensors and vectors are denoted using bold letters.

(a)

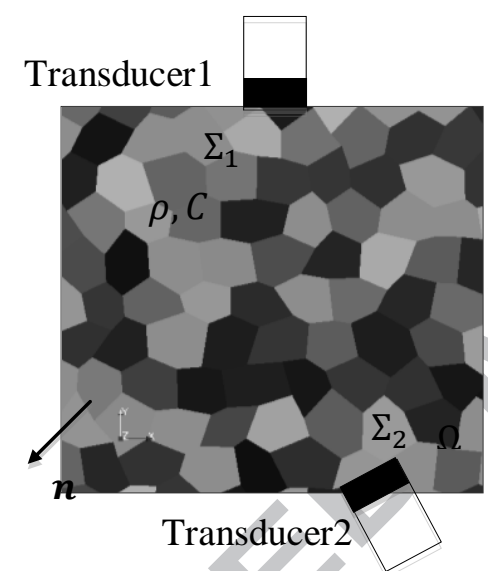

(b)

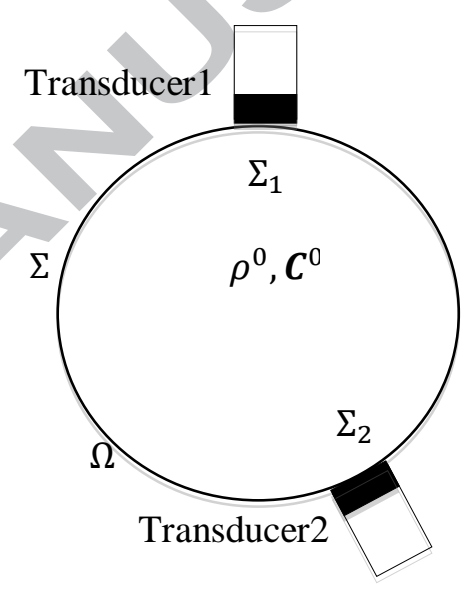

Fig. 1. Schematic diagram of the systems considered in the measurement of scattering in (a) a heterogeneous medium by comparison with (b) a homogeneous reference medium

Based on the reciprocity theorem, the basic idea of evaluating the grain-noise scattering is to quantify the so-called "reciprocity gap", which is derived by the comparison between the studied heterogeneous domain and the same domain but filled by a reference homogenous material. Denoting the elastic stiffness tensor and the density of the reference homogeneous material as $\boldsymbol{C}^{0}$ and $\rho^{0}$, the time harmonic elastic wave equation of the reference medium reads as:

$$
\operatorname{Div}_{\boldsymbol{x}} \boldsymbol{\sigma}^{0}+\rho^{0} \omega^{2} \boldsymbol{u}^{0}=\mathbf{0}
$$

In the following, the superscript "0" always indicates a mechanical field obtained in the reference homogeneous medium. Then the following variational formulations are straightforward for both systems:

$$
\begin{aligned}
& \left(\boldsymbol{\sigma}, \boldsymbol{\varepsilon}\left(\boldsymbol{u}^{0}\right)\right)_{\Omega}-\left(\rho \omega^{2} \boldsymbol{u}, \boldsymbol{u}^{0}\right)_{\Omega}=\left(\boldsymbol{\sigma} \cdot \boldsymbol{n}, \boldsymbol{u}^{0}\right)_{\partial \Omega} \\
& \left(\boldsymbol{\sigma}^{0}, \boldsymbol{\varepsilon}(\boldsymbol{u})\right)_{\Omega}-\left(\rho^{0} \omega^{2} \boldsymbol{u}^{0}, \boldsymbol{u}\right)_{\Omega}=\left(\boldsymbol{\sigma}^{0} \cdot \boldsymbol{n}, \boldsymbol{u}\right)_{\partial \Omega}
\end{aligned}
$$


where $\boldsymbol{n}$ is the outward unit normal to the boundary $\partial \Omega,(r, \cdot)_{\Omega}$ and $(\because,)_{\partial \Omega}$ denote integrations respectively over the volume $\Omega$ and the surface $\partial \Omega$ of appropriate dot product between two vectors or two tensors. Subtracting (4) from (3) leads to the following general equation of the reciprocity theorem for elastic waves in the presence of scatterers, such as grain boundaries:

$$
\left(\boldsymbol{\sigma} \cdot \boldsymbol{n}, \boldsymbol{u}^{0}\right)_{\partial \Omega}-\left(\boldsymbol{\sigma}^{0} \cdot \boldsymbol{n}, \boldsymbol{u}\right)_{\partial \Omega}=\left(\delta \boldsymbol{C}: \boldsymbol{\varepsilon}(\boldsymbol{u}), \boldsymbol{\varepsilon}\left(\boldsymbol{u}^{0}\right)\right)_{\Omega}-\omega^{2}\left(\delta \rho \boldsymbol{u}, \boldsymbol{u}^{0}\right)_{\Omega}
$$

with $\delta \boldsymbol{C}(\boldsymbol{x})=\boldsymbol{C}(\boldsymbol{x})-\boldsymbol{C}^{0}, \delta \rho(\boldsymbol{x})=\rho(\boldsymbol{x})-\rho^{0}$. It is obvious that, if there is no scatterers, i.e. $\delta \boldsymbol{C}=\mathbf{0}$ and $\delta \rho=0$, the terms on both sides of (5) are equal to zero. Both terms represent a reciprocity gap and can be used to measure the degree of perturbation due to the heterogeneities in the studied medium. Furthermore the equation (5) points out that there are two ways to evaluate the reciprocity gap. The first one implies to evaluate an integration over the whole volume of the studied domain by using a Green's function approach under the Born approximation assumption and gives rise to a theoretical estimation of the scattering. The second one implies to calculate a surface integration involving only the mechanical quantities available on the boundary of the studied domain and therefore can be used for experimental measurements.

\subsection{Physical signification of the reciprocity theorem applied to microstructural scattering analysis}

A brief recall of the scattering formulas proposed by Kino [9] allows an interesting insight into how the reciprocity gap in the case of polycrystalline materials is precisely related to the amplitude of elastic wave noise signals scattered by the inhomogeneities of polycrystalline microstructure.

For both heterogeneous and homogeneous reference systems shown in Fig. 1, two transducers in contact with the boundary $\partial \Omega$ are considered and the contact areas are respectively denoted as $\Sigma_{1}$ and $\Sigma_{2}$. The transducer used as a transmitter can trigger elastic waves in $\Omega$ by applying prescribed displacements or surface loadings on its contact area and the elastic wave fields within $\Omega$ are assumed to be not perturbed by the transducers. On the remainder of the boundary, i.e. $\Sigma=\partial \Omega \backslash\left(\Sigma_{1} \cup \Sigma_{2}\right)$, the free boundary condition is assumed. At the surface $\Sigma_{m}$ of the transmitter $m(m=1,2)$, the displacement and stress vector fields in time domain can be described as:

$$
\boldsymbol{u}_{m}^{+}(\boldsymbol{x}, t)=A_{m}^{+} \boldsymbol{u}_{m}^{+}(\boldsymbol{x}) \exp (-i \omega t), \boldsymbol{\sigma}_{m}^{+}(\boldsymbol{x}, t) \cdot \boldsymbol{n}=A_{m}^{+} \boldsymbol{\sigma}_{m}^{+}(\boldsymbol{x}) \cdot \boldsymbol{n} \exp (-i \omega t)
$$

Here $\omega$ is a given angular frequency, the superscript " + " represents the fields associated to the transmitter and $A_{m}^{+}$is the amplitude of elastic waves triggered by the transducer $m$. We note that $\boldsymbol{u}_{m}^{+}(\boldsymbol{x})$ and $\boldsymbol{\sigma}_{m}^{+}(\boldsymbol{x}) \cdot \boldsymbol{n}$ are chosen so the associated power rate is equal to one.

Once the prescribed displacements or the surface loadings vanish, the transducers can also serve as a receiver by responding to the elastic wave propagating in the opposite direction. If we assume that the received displacements and stress vectors have exactly the same form as (6), they can be written in the following form: 


$$
\boldsymbol{u}_{m}^{-}(\boldsymbol{x}, t)=A_{m}^{-} \boldsymbol{u}_{m}^{+*}(\boldsymbol{x}) \exp (-i \omega t), \boldsymbol{\sigma}_{m}^{-}(\boldsymbol{x}, t) \cdot \boldsymbol{n}=-A_{m}^{-} \boldsymbol{\sigma}_{m}^{+*}(\boldsymbol{x}) \cdot \boldsymbol{n} \exp (-i \omega t)
$$

where the superscript " * " indicates the complex conjugate, the superscript "-" represents the fields associated to the receiver. Since the systems considered here are linear, the displacement and the stress vector fields on the contact area of each transducer finally read as:

$$
\boldsymbol{u}_{m}(\boldsymbol{x})=A_{m}^{+} \boldsymbol{u}_{m}^{+}(\boldsymbol{x})+A_{m}^{-} \boldsymbol{u}_{m}^{+*}(\boldsymbol{x}), \boldsymbol{\sigma}_{m}(\boldsymbol{x}) \cdot \boldsymbol{n}=A_{m}^{+} \boldsymbol{\sigma}_{m}^{+}(\boldsymbol{x}) \cdot \boldsymbol{n}-A_{m}^{-} \boldsymbol{\sigma}_{m}^{+*}(\boldsymbol{x}) \cdot \boldsymbol{n}
$$

Then the scattering matrix $[S]=\left[S^{0}\right]+[\delta S]$ that contains ratios of received wave amplitudes to incident wave amplitudes is defined as:

$$
\left(\begin{array}{l}
A_{1}^{-} \\
A_{2}^{-}
\end{array}\right)=\left(\left[\begin{array}{ll}
S_{11}^{0} & S_{12}^{0} \\
S_{21}^{0} & S_{22}^{0}
\end{array}\right]+\left[\begin{array}{ll}
\delta S_{11} & \delta S_{12} \\
\delta S_{21} & \delta S_{22}
\end{array}\right]\right)\left(\begin{array}{l}
A_{1}^{+} \\
A_{2}^{+}
\end{array}\right)
$$

where $\left[S^{0}\right]$ is the scattering matrix of the homogeneous reference medium. Considering the interchangeability of the transmitter and the receiver, the reciprocity relation simply reads as $S_{21}^{0}=$ $S_{12}^{0}$. Herein only the microstructural noise scattering matrix $[\delta S]$ is of interest. Each term of $[\delta S]$ can be determined by different experimental settings.

We consider as an example a typical situation: Transducer 1 used as transmitter and Transducer 2 used as receiver for the heterogeneous medium for which the solutions are denoted as $\left(\boldsymbol{u}^{1 \rightarrow 2}, \boldsymbol{\sigma}^{1 \rightarrow 2}\right)$; Transducer 1 used as receiver and Transducer 2 used as transmitter for the reference medium for which the solutions are denoted as $\left(\boldsymbol{u}^{0 ; 2 \rightarrow 1}, \boldsymbol{\sigma}^{0 ; 2 \rightarrow 1}\right)$. In this situation the amplitudes of the elastic wave signals received or emitted by the transducers in both systems can be written as:

$$
\begin{gathered}
A_{1}^{+}=\text {finite, } A_{1}^{-}=0 ; A_{2}^{+}=0, A_{2}^{-}=\left(S_{21}^{0}+\delta S_{21}\right) A_{1}^{+} \\
\left(A_{1}^{+}\right)^{0}=0,\left(A_{1}^{-}\right)^{0}=S_{12}^{0}\left(A_{2}^{+}\right)^{0} ;\left(A_{2}^{+}\right)^{0}=\text { finite, }\left(A_{2}^{-}\right)^{0}=0
\end{gathered}
$$

Taking into account (10) and (11) and substituting the displacement and stress vector fields defined in (8) into the left-hand side of the reciprocity relation in (5), the amplitude of microstructural noise signals scattered by the polycrystalline medium can be calculated as follows:

$$
\begin{gathered}
\delta S_{21}(\omega)=\frac{-i \omega}{4 P_{0}(\omega)}\left(\left(\delta \boldsymbol{C}: \boldsymbol{\varepsilon}\left(\boldsymbol{u}^{1 \rightarrow 2}\right), \boldsymbol{\varepsilon}\left(\boldsymbol{u}^{0 ; 2 \rightarrow 1}\right)\right)_{\Omega}-\omega^{2}\left(\delta \rho \boldsymbol{u}^{1 \rightarrow 2}, \boldsymbol{u}^{0 ; 2 \rightarrow 1}\right)_{\Omega}\right) \\
\delta S_{21}(\omega)=\frac{-i \omega}{4 P_{0}(\omega)}\left(\left(\boldsymbol{\sigma}^{1 \rightarrow 2} \cdot \boldsymbol{n}, \boldsymbol{u}^{0 ; 2 \rightarrow 1}\right)_{\partial \Omega}-\left(\boldsymbol{\sigma}^{0 ; 2 \rightarrow 1} \cdot \boldsymbol{n}, \boldsymbol{u}^{1 \rightarrow 2}\right)_{\partial \Omega}\right)
\end{gathered}
$$

where $P_{0}(\omega)=A_{1}^{+}\left(A_{2}^{+}\right)^{0}$ denotes the incident power rate emitted by the transmitter.

Equations (12) and (13) express two equivalent ways to evaluate the microstructural noise scattering amplitude $\delta S_{21}$ using respectively a volume and a surface integrations. In Section 2.4, the equation (12) is used to obtain analytical formulas of the scattering coefficient based on an isolated scatterer model and using a Green's function approach, while the equation (13) allows the definition of our numerical procedure to measure the scattering coefficient using recorded mechanical data at the boundary of the studied domain in Section 2.6. Otherwise it is interesting to indicate that the scattered 
signal amplitude in any direction may be obtained by placing the two transducers 1 and 2 in the axis of that direction. For the particular case of the backscattering coefficient, which is the main object of the present work, the same formulas for the backscattering amplitude $\delta S_{11}$ are obtained by using two coincident transducers.

\subsection{Theoretical formulas of scattered amplitudes and differential cross sections based on isolated} scatterer model

The theoretical analysis of scattered amplitudes and differential cross sections proposed by Gubernatis et al. in 3D case [1,6] is recalled. As a new contribution, the development of the theoretical formulas in $2 \mathrm{D}$ case is given. The obtained 2D formulas are important for the comparison between the theoretical estimation and the 2D numerical simulations.

\subsubsection{Far-field scattered amplitudes}

The analysis based on an isolated scatterer model considers a bounded region of scatterers $\Omega_{\mathrm{sc}}$ embedded in an infinite homogeneous region $\mathrm{R}$ and calculates scattered amplitudes by using the farfield values of the scattered displacement and stress fields.

To do this, a coordinate system is adopted whose origin is set to be coincident with the centroid of the scatterer domain $\Omega_{\mathrm{sc}}$ (Fig. 2.).

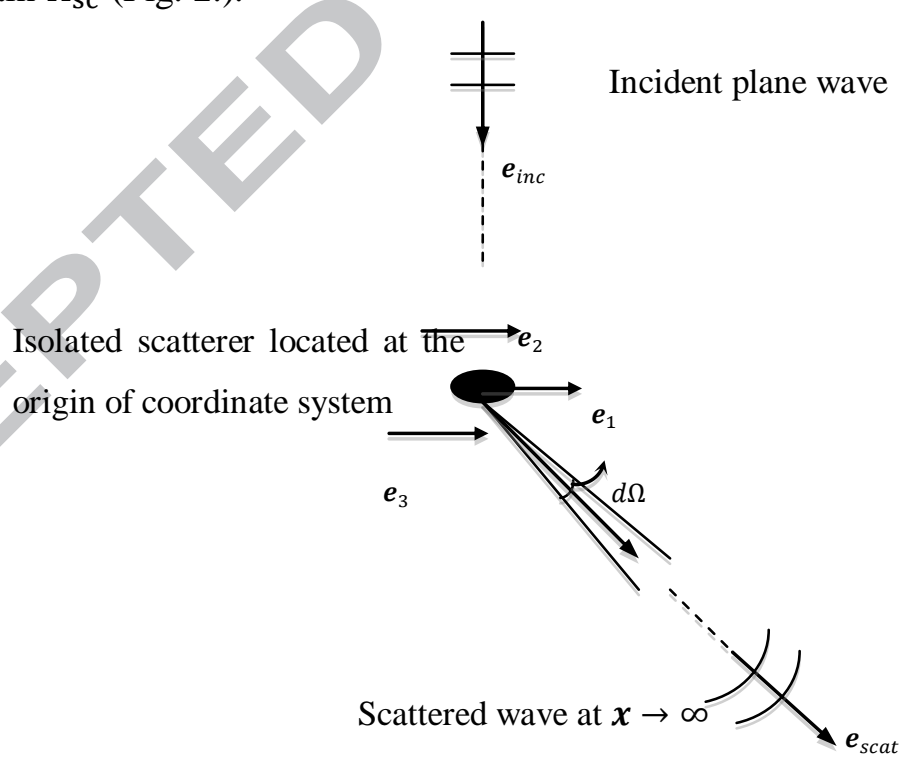

Fig. 2. Diagram of the systems considered in isolated scatter model (the 3D case)

In a very classical way, the displacement field in the whole region $\mathrm{R}$ is decomposed into two parts: the incident wave field $\boldsymbol{u}^{\text {inc }}$ and the scattered wave field $\boldsymbol{u}^{\text {scat }}$ :

$$
\boldsymbol{u}(\boldsymbol{x})=\boldsymbol{u}^{i n c}(\boldsymbol{x})+\boldsymbol{u}^{\text {scat }}(\boldsymbol{x})
$$

Then according to the well-known Green's function approach, the far field solution of the scattered displacement $\boldsymbol{u}^{\text {scat }}$ for all $\boldsymbol{x} \in \mathrm{R}$ reads as: 


$$
\boldsymbol{u}^{\text {scat }}(\boldsymbol{x})=\int_{\Omega_{\mathrm{sc}}} \delta \rho \omega^{2} \boldsymbol{G}^{T}\left(0 ; \boldsymbol{x}^{\prime}-\boldsymbol{x}\right) \cdot \boldsymbol{u}\left(\boldsymbol{x}^{\prime}\right) d \boldsymbol{x}^{\prime}+\int_{\Omega_{\mathrm{sc}}}\left(\boldsymbol{\varepsilon}\left(\boldsymbol{u}\left(\boldsymbol{x}^{\prime}\right)\right): \delta \boldsymbol{C}: \boldsymbol{\varepsilon}\left(\boldsymbol{G}\left(0 ; \boldsymbol{x}-\boldsymbol{x}^{\prime}\right) \cdot \boldsymbol{e}_{m}\right)\right) \boldsymbol{e}_{m} d \boldsymbol{x}^{\prime}(15)
$$

where $\left\{\boldsymbol{e}_{m}\right\}_{m=1,2,3}$ denotes the global Cartesian basis. We note that the farfield assumption allows replacing $\boldsymbol{G}\left(\boldsymbol{x}^{\prime} ; \boldsymbol{x}\right)$, the Green's function tensor with $\boldsymbol{x}^{\prime}$ as the source point, by $\boldsymbol{G}\left(0 ; \boldsymbol{x}-\boldsymbol{x}^{\prime}\right)$.

It is obvious that the equation (15) expressing the scattered wave field in terms of an integration over the volume of the scatter region $\Omega_{\mathrm{sc}}$ cannot be directly used, as the displacement field $\boldsymbol{u}(\boldsymbol{x})$ within $\Omega_{\mathrm{sc}}$ is generally unknown. However it is useful to develop a theoretical estimation of the scattered amplitudes when the Green's function tensor $\boldsymbol{G}\left(0 ; \boldsymbol{x}-\boldsymbol{x}^{\prime}\right)$ is known. It is precisely the case for an infinite isotropic and homogeneous elastic domain.

In $3 \mathrm{D}$ or $2 \mathrm{D}$ infinite isotropic and homogeneous elastic media, with $\lambda^{0}$ and $\mu^{0}$ as Lamé parameters and $\rho^{0}$ as density, the Green's function tensor reads as [25]:

$$
\begin{gathered}
\boldsymbol{G}^{3 D}(0 ; \boldsymbol{r})=\frac{1}{4 \pi \rho^{0} \omega^{2}}\left(\boldsymbol{\nabla}_{\boldsymbol{r}}\left(\boldsymbol{\nabla}_{\boldsymbol{r}}\left(\frac{\exp \left(i k_{0 S} r\right)}{r}-\frac{\exp \left(i k_{0 L} r\right)}{r}\right)\right)+k_{0 S}^{2} \frac{\exp \left(i k_{0 S} r\right)}{r} \mathbf{I}^{3 \mathrm{D}}\right) \\
\boldsymbol{G}^{2 D}(0 ; \boldsymbol{r})=\frac{i}{4 \rho^{0} \omega^{2}}\left(\boldsymbol{\nabla}_{\boldsymbol{r}}\left(\boldsymbol{\nabla}_{\boldsymbol{r}}\left(H_{0}^{(1)}\left(k_{0 S} r\right)-H_{0}^{(1)}\left(k_{0 L} r\right)\right)\right)+k_{0 S}^{2} H_{0}^{(1)}\left(k_{0 S} r\right) \mathbf{I}^{2 \mathrm{D}}\right)
\end{gathered}
$$

with $\boldsymbol{r}=\boldsymbol{x}-\boldsymbol{x}^{\prime}, r=|\boldsymbol{r}|$, I the 3D or 2D identity matrix, $v_{0 L}=\sqrt{\left(\lambda^{0}+2 \mu^{0}\right) / \rho^{0}}$ and $v_{0 S}=\sqrt{\mu^{0} / \rho^{0}}$ respectively the phase velocities of the longitudinal and the shear waves in the unperturbed medium, and $k_{0 L}=\omega / v_{0 L}$ and $k_{0 S}=\omega / v_{0 S}$ respectively the corresponding wave numbers. For the $2 \mathrm{D}$ case, $H_{0}^{(1)}(\cdot)$ denotes the Hankel function of the first kind, which expresses the outward-propagating cylindrical wave solutions since it is assumed to be uniform along the direction perpendicular to the plane of the $2 \mathrm{D}$ problem.

Denoting by $\boldsymbol{e}_{\text {scat }}(\boldsymbol{x})$ the considered unit scattered direction vector, i.e. $\boldsymbol{x}=|\boldsymbol{x}| \boldsymbol{e}_{\text {scat }}$, and taking into account the fact that, for $\boldsymbol{x}^{\prime} \in \Omega_{\mathrm{sc}}$ and $\boldsymbol{x} \in \mathrm{R}$, we have $r^{-1} \sim|\boldsymbol{x}|^{-1}$ and $\boldsymbol{r} \sim|\boldsymbol{x}|-\boldsymbol{x}^{\prime} \cdot \boldsymbol{e}_{\text {scat }}$ when $|x| \rightarrow \infty$, the far-field solution of the scattered displacement $\boldsymbol{u}^{\text {scat }}$ and stress $\boldsymbol{\sigma}^{\text {scat }}$ can be derived from (15) as follows:

$$
\begin{gathered}
\boldsymbol{u}^{s c a t}(\boldsymbol{x}) \sim \sum_{l=L, S} \boldsymbol{A}_{l}\left(\boldsymbol{e}_{s c a t}\right) \frac{\exp \left(i k_{0 l}|x|\right)}{|\boldsymbol{x}|^{(N} \operatorname{dim}^{-1) / 2}} \\
\boldsymbol{\sigma}^{s c a t}(\boldsymbol{x}) \sim i \lambda k_{0 L}\left(\boldsymbol{A}_{L} \cdot \boldsymbol{e}_{s c a t}\right) \frac{\exp \left(i k_{0 L}|\boldsymbol{x}|\right)}{|\boldsymbol{x}|^{\left({ }^{(N i m}-1\right) / 2}} \mathbf{I}+2 i \mu \sum_{l=L, S} k_{0 l}\left(\boldsymbol{A}_{l} \otimes_{S} \boldsymbol{e}_{s c a t}\right) \frac{\exp \left(i k_{0 l}|\boldsymbol{x}|\right)}{|\boldsymbol{x}|^{\left(N_{d i m}-1\right) / 2}}
\end{gathered}
$$

with

$$
\begin{gathered}
\boldsymbol{A}_{L}\left(\boldsymbol{e}_{s c a t}\right)=\left(\boldsymbol{e}_{s c a t} \otimes \boldsymbol{e}_{s c a t}\right) \cdot \boldsymbol{a}\left(\boldsymbol{k}_{0 L}\right) \\
\boldsymbol{A}_{S}\left(\boldsymbol{e}_{s c a t}\right)=\left(\mathbf{I}-\boldsymbol{e}_{s c a t} \otimes \boldsymbol{e}_{s c a t}\right) \cdot \boldsymbol{a}\left(\boldsymbol{k}_{0 S}\right) \\
\boldsymbol{a}\left(\boldsymbol{k}_{0 l}\right)=\frac{\chi\left(k_{0 l}, N_{d i m}\right)}{4 \pi \rho^{0}\left(v_{0 l}\right)^{2}} \int_{\Omega_{s c}}\left(\omega^{2} \delta \rho\left(\boldsymbol{x}^{\prime}\right) \boldsymbol{u}\left(\boldsymbol{x}^{\prime}\right)-i \boldsymbol{k}_{0 l} \cdot \delta \boldsymbol{C}\left(\boldsymbol{x}^{\prime}\right): \boldsymbol{\varepsilon}\left(\boldsymbol{u}\left(\boldsymbol{x}^{\prime}\right)\right) \exp \left(-i \boldsymbol{k}_{0 l} \cdot \boldsymbol{x}^{\prime}\right) d \boldsymbol{x}^{\prime}\right.
\end{gathered}
$$


where the symbol " $\sim$ " indicates the asymptotic forms, $\otimes_{s}$ denotes the symmetric tensor product defined as: $\boldsymbol{a} \otimes_{S} \boldsymbol{b}=\frac{1}{2}(\boldsymbol{a} \otimes \boldsymbol{b}+\boldsymbol{b} \otimes \boldsymbol{a})$ with $\otimes$ denoting the usual tensor product, $\boldsymbol{k}_{0 L}=k_{0 L} \boldsymbol{e}_{\text {scat }}$ and $\boldsymbol{k}_{0 S}=k_{0 S} \boldsymbol{e}_{s c a t}$ are the wave vectors of respectively the longitudinal waves and the shear waves.

To gather the formulas in both $2 \mathrm{D}$ and $3 \mathrm{D}$ cases, we propose in the present work to introduce a space dimension dependent constant $\chi\left(k_{0 l}, N_{\text {dim }}\right)$ :

$$
\chi\left(k_{0 l}, N_{\text {dim }}\right)=\left\{\begin{array}{l}
1, \quad N_{\text {dim }}=3 \\
\frac{(1+i) \sqrt{\pi}}{\sqrt{k_{0 l}}}, N_{\text {dim }}=2
\end{array}\right.
$$

Equation (18) shows that the scattered field $\boldsymbol{u}^{\text {scat }}$ can be decomposed into a longitudinal wave contribution with amplitude $\boldsymbol{A}_{L}$ parallel to $\boldsymbol{e}_{\text {scat }}$ and a shear wave contribution with amplitude $\boldsymbol{A}_{S}$ perpendicular to $\boldsymbol{e}_{\text {scat }}$. Both amplitudes are determined by the vectors $\left(\boldsymbol{k}_{0 l}\right)(l=L, S)$, whose complete calculation requires the complete information of the field $\boldsymbol{u}$ inside $\Omega_{\mathrm{sc}}$.

\subsubsection{Theoretical formulas of differential cross sections}

Classically the differential cross section $\frac{d P(\omega)}{d \Omega}\left(\boldsymbol{e}_{\text {scat }}\right)$ gives, for a given angular frequency $\omega$, the measure of the fraction of incident power scattered into a particular direction $\boldsymbol{e}_{\text {scat }}$ across the surface $d A$ defined by the differential element of solid angle $d \Omega$ in that direction. As $d A=|\boldsymbol{x}|^{N_{d i m}-1} d \Omega$, it can be proved that:

$$
\frac{d P(\omega)}{d \Omega}\left(\boldsymbol{e}_{\text {scat }}\right)=\lim _{|x| \rightarrow \infty}\left(-\frac{\omega}{2} \operatorname{Im}\left(|x|^{N_{\text {dim }}-1}\left(\boldsymbol{\sigma}^{\text {scat }} \cdot \boldsymbol{e}_{\text {scat }}\right) \cdot \boldsymbol{u}^{\text {scat } *}\right)\right) / P_{0}(\omega)
$$

where $P_{0}(\omega)$ is the power rate of the incident fields. When $|\boldsymbol{x}| \rightarrow \infty$, as $\boldsymbol{\sigma}^{\text {scat }}$ and $\boldsymbol{u}^{\text {scat }}$ are respectively proportional to $|\boldsymbol{x}|^{-\left(N_{d i m}-1\right) / 2}$ (see (18), (19)), the equation of the differential cross section (24) shows that it is independent of the distance from the scatterer.

In the case of an incident field of incident direction $\boldsymbol{e}_{i n c}$ and containing both longitudinal and shear waves of amplitude $\boldsymbol{A}_{i L}$ and $\boldsymbol{A}_{i S}$ defined in the following way:

$$
\boldsymbol{u}^{i n c}(\boldsymbol{x})=\boldsymbol{A}_{i L} \exp \left(i k_{0 L} \boldsymbol{e}_{i n c} \cdot \boldsymbol{x}-i \omega t\right)+\boldsymbol{A}_{i S} \exp \left(i k_{0 S} \boldsymbol{e}_{i n c} \cdot \boldsymbol{x}-i \omega t\right)
$$

It can be shown that the differential cross sections for both 3D and 2D cases have the same expression as follows:

$$
\frac{d P(\omega)}{d \Omega}\left(\boldsymbol{e}_{\text {scat }}\right)=\frac{k_{0 L}\left(\lambda^{0}+2 \mu^{0}\right)\left|\boldsymbol{A}_{L}\left(\boldsymbol{e}_{\text {scat }}\right)\right|^{2}+\mu^{0} k_{0 S}\left|\boldsymbol{A}_{S}\left(\boldsymbol{e}_{\text {scat }}\right)\right|^{2}}{k_{0 L}\left(\lambda^{0}+2 \mu^{0}\right)\left|\boldsymbol{A}_{i L}\right|^{2}+\mu^{0} k_{0 S}\left|\boldsymbol{A}_{i S}\right|^{2}}
$$

So the scattered amplitudes are directly linked to the differential cross sections.

In the case where the incident field contains only longitudinal waves, i.e. $\left|\boldsymbol{A}_{i s}\right|=0$, we have:

$$
\frac{d P(\omega)}{d \Omega}\left(\boldsymbol{e}_{\text {scat }}\right)=\frac{\left|\boldsymbol{A}_{L}\left(\boldsymbol{e}_{\text {scat }}\right)\right|^{2}}{\left|A_{i L}\right|^{2}}+\frac{v_{0 S}}{v_{0 P}} \frac{\left|A_{S}\left(\boldsymbol{e}_{s c a t}\right)\right|^{2}}{\left|A_{i L}\right|^{2}}
$$


The two terms on the right hand side of (27) are respectively longitudinal-to-longitudinal and longitudinal-to-shear differential cross sections. In the following, only the longitudinal-to-longitudinal differential cross section $\frac{\left|A_{L}\left(\boldsymbol{e}_{\text {scat }}\right)\right|^{2}}{\left|\boldsymbol{A}_{i L}\right|^{2}}$ is considered in the theoretical analysis.

\subsection{Theoretical evaluations of microstructural noise scattering coefficient}

The microstructural noise scattering effectiveness is quantified by the scattering coefficient $\eta\left(\omega, \boldsymbol{e}_{\text {scat }}\right)$, defined as the differential scattering cross section per unit volume for a given angular frequency $\omega$ and in a particular direction $\boldsymbol{e}_{\text {scat }}$ per unit incident power rate. Sections 2.2 and 2.3 present two ways to evaluate $\eta\left(\omega, \boldsymbol{e}_{\text {scat }}\right)$ and we recall in the present section the equivalence between them under the assumption of weak-scattering and with the use of the Born approximation. Furthermore, the comparison of both methods allows the identification of a proportionality coefficient between them, already given in the literature for the 3D case. Then as a new result this proportionality coefficient is identified in the $2 \mathrm{D}$ case.

Now we consider the polycrystalline domain $\Omega$ by assuming that it is single phase with randomly oriented grains and is macroscopically isotropic and homogeneous. We denote by $N$ the total number of grains in the active volume $V_{p c}$ of grain scattering [10]. The characteristic function $\gamma^{I}$ for the $I^{\text {th }}$ grain $g^{I}$ is equal to one inside $g^{I}$ and to zero otherwise [10]. It is assumed that there is no deviation of density from one grain to the other and the deviation of elastic tensor $\delta \boldsymbol{C}(x)$ in each grain is constant and is denoted as $\delta \boldsymbol{C}^{I}$ for the grain $g^{I}$.

By the first method based on the independent scattering approximation, each grain in $\Omega$ is regarded as an isolated scatterer placed in the homogeneous reference medium and their contributions to the total scattering are summed up incoherently. This gives rise to the following formula of the scattering coefficient:

$$
\eta\left(\omega, \boldsymbol{e}_{s c a t}\right)=\frac{1}{\left|\boldsymbol{A}_{i L}\right|^{2}} \frac{N}{V_{p c}}\left\langle\left|\boldsymbol{A}_{L}\left(g^{I}, \boldsymbol{e}_{\text {scat }}\right)\right|^{2}\right\rangle=\frac{1}{\left|\boldsymbol{A}_{i L}\right|^{2}} \frac{N}{V_{p c}} \frac{\sum_{I}\left|\boldsymbol{A}_{L}\left(g^{I}, \boldsymbol{e}_{\text {scat }}\right)\right|^{2}}{N}
$$

where $\langle$.$\rangle denotes an average over all the N$ grains. As previously mentioned, the longitudinal-to-shear scattering part is neglected in (28).

Due to the single phase assumption and when $N$ is large enough, it is obvious that the average $\left\langle\delta \boldsymbol{C}^{I} \otimes \delta \boldsymbol{C}^{I}\right\rangle$ over all grains is equal to the average over all crystallographic orientations, denoted simply by $\left\langle\delta \boldsymbol{C}^{\mathrm{g}} \otimes \delta \boldsymbol{C}^{\mathrm{g}}\right\rangle$. According to the far-field scattering amplitude presented in Section 2.3 and using the Born approximation by replacing in (20)-(22) the scattered field $\boldsymbol{u}^{I}(\boldsymbol{x})$ by each grain $g^{I}$ by the unperturbed field $\boldsymbol{u}^{i n c}(\boldsymbol{x})$ in the homogeneous medium, the scattering coefficient can finally be written as:

$$
\begin{gathered}
\eta\left(\omega, \boldsymbol{e}_{s c a t}\right)\left(\frac{1}{V_{p c}} \frac{\left|\chi\left(k_{0 L}, N_{d i m}\right)\right|^{2}}{\left(4 \pi \rho^{0} v_{0 L}^{2}\right)^{2}\left|\boldsymbol{A}_{i L}\right|^{2}}\right)^{-1}= \\
\sum_{I} \int_{\Omega} d \boldsymbol{x} \int_{\Omega} d \boldsymbol{x}^{\prime} k_{0 P}^{2}\left\langle\gamma^{I}(\boldsymbol{x}) \gamma^{I}\left(\boldsymbol{x}^{\prime}\right)\right\rangle \boldsymbol{E}_{1}(\boldsymbol{x}):\left\langle\delta \boldsymbol{C}^{\mathrm{g}} \otimes \delta \boldsymbol{C}^{\mathrm{g}}\right\rangle: \boldsymbol{E}_{1}^{*}\left(\boldsymbol{x}^{\prime}\right) \exp \left(-i k_{0 L} \boldsymbol{e}_{s c a t} \cdot\left(\boldsymbol{x}-\boldsymbol{x}^{\prime}\right)\right)
\end{gathered}
$$


with $\boldsymbol{E}_{1}(\cdot)=\boldsymbol{e}_{\text {scat }} \otimes \boldsymbol{e}_{\text {scat }} \otimes \boldsymbol{\varepsilon}\left(\boldsymbol{u}^{\text {inc }}(\cdot)\right)$ and $\left\langle\gamma^{I}(\boldsymbol{x}) \gamma^{I}\left(\boldsymbol{x}^{\prime}\right)\right\rangle$ the spatial correlation function of two points $\boldsymbol{x}$ and $\boldsymbol{x}^{\prime}$, which quantifies the possibility that they belong to the same grain $g^{I}$.

The second method proposed by Rose [10] is based on Auld's reciprocity theorem and states that $\eta\left(\omega, \boldsymbol{e}_{\text {scat }}\right)$ can be obtained from the scattered noise signal amplitude $\delta S(\omega)$ defined by (12) in the following way:

$$
\eta\left(\omega, \boldsymbol{e}_{s c a t}\right)=\frac{\beta\left\langle\delta S(\omega) \delta S^{*}(\omega)\right\rangle}{V_{p c}}
$$

where $\langle$.$\rangle is an average over all samples, \beta$ is a coefficient of proportionality and can be determined by the equivalence between the two methods (28) and (30) by considering some particular cases. It is important to recall that for this method, it is required that $\langle\delta S(\omega)\rangle=0$, so $\eta\left(\omega, \boldsymbol{e}_{\text {scat }}\right)$ can be considered as the variance measure of noises. This requirement leads to $\langle\delta \boldsymbol{C}(\boldsymbol{x})\rangle=0$ and implies that we should use the Voigt average of the elastic tensor, denoted by $\langle\delta \boldsymbol{C}(\boldsymbol{x})\rangle^{\text {Voigt }}$, as the stiffness tensor for the reference medium.

By substituting (12) into (30) and by noticing that $\delta \rho=0$, we obtain:

$$
\eta\left(\omega, \boldsymbol{e}_{s c a t}\right)\left(\frac{\beta}{V_{p c}}\left(\frac{\omega}{4 P_{0}(\omega)}\right)^{2}\right)^{-1}=\int_{\Omega} d \boldsymbol{x} \int_{\Omega} d \boldsymbol{x}^{\prime} \boldsymbol{E}_{2}(\boldsymbol{x}):\left\langle\delta \boldsymbol{C}(\boldsymbol{x}) \otimes \delta \boldsymbol{C}\left(\boldsymbol{x}^{\prime}\right)\right\rangle: \boldsymbol{E}_{2}^{*}\left(\boldsymbol{x}^{\prime}\right)
$$

with $\boldsymbol{E}_{2}(\cdot)=\boldsymbol{\varepsilon}\left(\boldsymbol{u}^{1 \rightarrow 2}(\boldsymbol{x})\right) \otimes \boldsymbol{\varepsilon}\left(\boldsymbol{u}^{0 ; 2 \rightarrow 1}(\cdot)\right)$. The term $\delta \boldsymbol{C}(\boldsymbol{x}) \otimes \delta \boldsymbol{C}\left(\boldsymbol{x}^{\prime}\right)$ refers to the correlation function of elastic stiffness tensors at two arbitrary points $\boldsymbol{x}$ and $\boldsymbol{x}^{\prime}$.

As $\delta \boldsymbol{C}(\boldsymbol{x})=\sum_{I} \delta \boldsymbol{C}^{I} \gamma^{I}(\boldsymbol{x})$, by assuming that the elastic constant variation and the characteristic functions vary independently on the one hand, and on the other hand the deviation in the elastic constants vary independently from grain to grain, i.e. $\left\langle\delta \boldsymbol{C}^{I} \otimes \delta \boldsymbol{C}^{J}\right\rangle=0$ when $I \neq J$, we can write:

$$
\left\langle\delta \boldsymbol{C}(\boldsymbol{x}) \otimes \delta \boldsymbol{C}\left(\boldsymbol{x}^{\prime}\right)\right\rangle=\sum_{I} \sum_{J}\left\langle\delta \boldsymbol{C}^{I} \otimes \delta \boldsymbol{C}^{J}\right\rangle\left\langle\gamma^{\mathrm{I}}(\boldsymbol{x}) \gamma^{\mathrm{J}}\left(\boldsymbol{x}^{\prime}\right)\right\rangle=\left\langle\delta \boldsymbol{C}^{\mathrm{g}} \otimes \delta \boldsymbol{C}^{\mathrm{g}}\right\rangle \sum_{I}\left\langle\gamma^{I}(\boldsymbol{x}) \gamma^{I}\left(\boldsymbol{x}^{\prime}\right)\right\rangle
$$

Therefore we finally obtain:

$\eta\left(\omega, \boldsymbol{e}_{\text {scat }}\right)\left(\frac{\beta}{V_{p c}}\left(\frac{\omega}{4 P_{0}(\omega)}\right)^{2}\right)^{-1}=\sum_{I} \int_{\Omega} d \boldsymbol{x} \int_{\Omega} d \boldsymbol{x}^{\prime}\left\langle\gamma^{I}(\boldsymbol{x}) \gamma^{I}\left(\boldsymbol{x}^{\prime}\right)\right\rangle \boldsymbol{E}_{\mathbf{2}}(\boldsymbol{x}):\left\langle\delta \boldsymbol{C}^{\mathrm{g}} \otimes \delta \boldsymbol{C}^{\mathrm{g}}\right\rangle: \boldsymbol{E}_{\mathbf{2}}^{*}\left(\boldsymbol{x}^{\prime}\right)$

Once more, when the Born approximation is used if the elastic constant deviation is small and the weak-scattering assumption is appropriate, the unknown fields $\boldsymbol{u}^{1 \rightarrow 2}$ in (33) is replaced by $\boldsymbol{u}^{0 ; 1 \rightarrow 2}$ the solution of the same problem for $\boldsymbol{u}^{1 \rightarrow 2}$ but in the homogeneous reference medium. In the case of backscattering, the two transducers 1 and 2 coincide and we have furthermore $\boldsymbol{u}^{0 ; 1 \rightarrow 2}=\boldsymbol{u}^{0 ; 2 \rightarrow 1}$, then theoretical formulas of $\eta$ of backscattering can be obtained (see Section 2.5).

Finally to identify the constant of proportionality $\beta$, the equations (29) and (33) are compared under the Born approximation and in the particular case of backscattering with an incident wave that is assumed to be the following longitudinal plane wave with the propagating direction $\boldsymbol{e}_{\text {inc }}=-\boldsymbol{e}_{\text {scat }}$ and of amplitude $U_{L}^{0}$ : 


$$
\boldsymbol{u}^{\text {inc }}(\boldsymbol{x})=U_{L}^{0} \boldsymbol{e}_{\text {inc }} \exp \left(i k_{0 L} \boldsymbol{e}_{\text {inc }} \cdot \boldsymbol{x}\right)
$$

By denoting $\boldsymbol{E}_{\text {scat }}=\boldsymbol{e}_{\text {scat }} \otimes \boldsymbol{e}_{\text {scat }} \otimes \boldsymbol{e}_{\text {scat }} \otimes \boldsymbol{e}_{\text {scat }}, \quad$ it is straightforward that $\boldsymbol{E}_{1}(\cdot)=$ $i U_{L}^{0} k_{0 L} \boldsymbol{E}_{\text {scat }} \exp \left(i k_{0 L} \boldsymbol{e}_{\text {scat }} \cdot(\cdot)\right)$ and $\boldsymbol{E}_{2}(\cdot)=-\left(U_{L}^{0} k_{0 L}\right)^{2} \boldsymbol{E}_{\text {scat }} \exp \left(-2 i k_{0 L} \boldsymbol{e}_{\text {scat }} \cdot(\cdot)\right),(29)$ and (33) then respectively become:

$$
\eta\left(\omega, \boldsymbol{e}_{s c a t}\right)\left(\frac{1}{V_{p c}} \frac{\left|\chi\left(k_{0 L}, N_{d i m}\right)\right|^{2} k_{0 L}^{4}}{\left(4 \pi \rho^{0} v_{0 L}^{2}\right)^{2}}\right)^{-1}=
$$

$\left(\sum_{I} \int_{\Omega} d \boldsymbol{x} \int_{\Omega} d \boldsymbol{x}^{\prime}\left\langle\gamma^{I}(\boldsymbol{x}) \gamma^{I}\left(\boldsymbol{x}^{\prime}\right)\right\rangle \exp \left(-2 i k_{0 L} \boldsymbol{e}_{\text {scat }} \cdot\left(\boldsymbol{x}-\boldsymbol{x}^{\prime}\right)\right)\right) \boldsymbol{E}_{\text {scat }}:\left\langle\delta \boldsymbol{C}^{\mathrm{g}} \otimes \delta \boldsymbol{C}^{\mathrm{g}}\right\rangle: \boldsymbol{E}_{\text {scat }}$

$$
\eta\left(\omega, \boldsymbol{e}_{s c a t}\right)\left(\frac{\beta}{V_{p c}}\left(\frac{\omega}{4 P_{0}(\omega)}\right)^{2}\left(U_{L}^{0}\right)^{4} k_{0 L}^{4}\right)^{-1}=
$$

$\left(\sum_{I} \int_{\Omega} d \boldsymbol{x} \int_{\Omega} d \boldsymbol{x}^{\prime}\left\langle\gamma^{I}(\boldsymbol{x}) \gamma^{I}\left(\boldsymbol{x}^{\prime}\right)\right\rangle \exp \left(-2 i k_{0 L} \boldsymbol{e}_{s c a t} \cdot\left(\boldsymbol{x}-\boldsymbol{x}^{\prime}\right)\right)\right) \boldsymbol{E}_{\text {scat }}:\left\langle\delta \boldsymbol{C}^{\mathrm{g}} \otimes \delta \boldsymbol{C}^{\mathrm{g}}\right\rangle: \boldsymbol{E}_{\text {scat }}$

The comparison between (35) and (36) finally results in the following formula of $\beta$, which depend also on the space dimension $N_{\text {dim }}$ :

$$
\beta\left(\omega, N_{\text {dim }}\right)=\left(\frac{4 P_{0}(\omega)}{\omega}\right)^{2} \frac{1}{\left(U_{L}\right)^{2}\left(U_{L}^{0}\right)^{2}} \frac{\left|\chi\left(k_{0 L}, N_{d i m}\right)\right|^{2}}{\left(4 \pi \rho^{0} v_{0 L}^{2}\right)^{2}}
$$

where $\left(U_{L}^{0}\right)^{4}$ is replaced by $\left(U_{L}\right)^{2}\left(U_{L}^{0}\right)^{2}$ in order to preserve the generality when the Born approximation is not applied, especially for the formulas developed for the numerical approach presented in Section 2.6.

In the 3D case, (37) gives the same result obtained by Margetan et al. [11]. In the 2D case, it is necessary to multiply by $\left|\chi\left(k_{0 L}, N_{d i m}=2\right)\right|^{2}=\frac{2 \pi}{k_{0 L}}$, which is inversely proportional to the norm of the longitudinal wave vector $k_{0 L}$.

Finally introducing (37) in (30), we obtained the following formula of the scattering coefficient based on the reciprocity theorem in general cases, which is used to define our numerical measure strategy (Section 2.6):

$$
\eta\left(\omega, \boldsymbol{e}_{s c a t}\right)=\frac{1}{V_{p c}} \frac{1}{\left(U_{L}\right)^{2}\left(U_{L}^{0}\right)^{2}} \frac{\left|\chi\left(k_{0 L}, N_{d i m}\right)\right|^{2}}{\left(4 \pi \rho^{0} v_{0 L}^{2}\right)^{2}}\left(\frac{4 P_{0}(\omega)}{\omega}\right)^{2}\left\langle\delta S(\omega) \delta S^{*}(\omega)\right\rangle
$$

\section{5 $2 D$ and $3 D$ analytical results for grain size effects on ultrasonic backscattering coefficient}

In this section, we recall the analytical formulas of the backscattering coefficient for a singlephase, untextured and equiaxed polycrystalline medium without density variation in the 3D case and develop it in the $2 \mathrm{D}$ case by applying the theoretical results previously presented.

For ease of analysis, it is assumed that the wave vector of the incident wave is in the direction $\boldsymbol{e}_{i n c}=-\boldsymbol{e}_{2}$. When the backscattering is considered, we have $\boldsymbol{e}_{\text {scat }}=-\boldsymbol{e}_{\text {inc }}$. As only the longitudinalto-longitudinal scattering is considered, the incident wave is assumed to be a longitudinal wave. The longitudinal-to-longitudinal backscattering coefficient is simply denoted as $\eta(\omega)$. 
Previous research works [2] indicate that the two-point spatial correlation function measures the probability that two points $\boldsymbol{x}$ and $\boldsymbol{x}^{\prime}$ belong to the same grain. For a common model of randomlyplaced and equally sized spherical grains, it is often approximated by an exponential function depending on the distance between the two points $r=\left|\boldsymbol{x}-\boldsymbol{x}^{\prime}\right|$ :

$$
\sum_{I}\left\langle\gamma^{\mathrm{I}}(\boldsymbol{x}) \gamma^{\mathrm{I}}\left(\boldsymbol{x}^{\prime}\right)\right\rangle=\exp (-2 r / \bar{d})
$$

where $\bar{d}$ is the averaged grain diameter. Then by substituting (39) into (35) and by applying the following change of variables:

$$
s=\left(x+x^{\prime}\right) / 2, r=x-x^{\prime}
$$

the following equation is straightforward:

$$
\eta(\omega)=\frac{\left|\chi\left(k_{0 L}, N_{d i m}\right)\right|^{2}}{\left(4 \pi \rho^{0} v_{0 L}^{2}\right)^{2}}\left(k_{0 L}\right)^{4}\left\langle\delta C_{2222} \delta C_{2222}\right\rangle \int_{\mathrm{R}} d \boldsymbol{r}\left(\exp \left(i k_{0 L} \boldsymbol{r} . \boldsymbol{e}_{2}\right) \exp \left(-\frac{2 r}{\bar{d}}\right)\right)
$$

Then considering the integration form (41) in the spherical coordinate and the cylindrical coordinate systems respectively for 3D and 2D cases, the analytical formulas for the longitudinal-tolongitudinal backscattering coefficient are obtained:

$$
\begin{aligned}
& \eta^{3 D}(\omega)=\frac{\left\langle\delta C_{2222} \delta C_{2222}\right\rangle}{\left(4 \pi \rho^{0}\left(v_{0 L}\right)^{2}\right)^{2}} \frac{\pi\left(x_{0}\right)^{4}}{\left(1+\left(x_{0}\right)^{2}\right)^{2} \bar{d}} \\
& \eta^{2 D}(\omega)=\pi \sqrt{\left(x_{0}\right)^{-2}+1} \eta^{3 D}(\omega)
\end{aligned}
$$

They are consistent with the results given by Rose [10] and Ghoshal et al. [20], respectively. Here $x_{0}$ measures the ratio of the averaged grain size to the wavelength $\lambda_{0 L}$ and is referred as "normalized frequency":

$$
x_{0}=k_{0 L} \bar{d}=\frac{2 \pi \bar{d}}{\lambda_{0 L}}
$$

Concerning the correlation function of the elastic stiffness tensor, only $\left\langle\delta C_{2222} \delta C_{2222}\right\rangle$ is needed in the present work and is calculated using the following equation proposed in [7] for the case of macroscopically isotropic and homogeneous polycrystals composed of single phase cubic symmetric crystallites:

$$
\left\langle\delta C_{2222} \delta C_{2222}\right\rangle=48\left(a_{c}\right)^{2} / 1575
$$

with $a_{c}=C_{1111}-C_{1122}-2 C_{2323}$ the invariant anisotropy factor for cubic symmetry crystallites. Particular attention should be paid to the single-scattering assumption and the Born approximation used in the derivation of analytical expressions for the backscattering coefficient. They are expected to be good approximations on the condition that the inhomogeneity degree of polycrystals $\epsilon$ is much less than unity, which can be expressed in terms of the effective elastic constants as proposed in [2]: 


$$
\left(\epsilon_{i j k l}\right)^{2} \cong \frac{1}{4} \frac{\left\langle\left[C_{i j k l}(\boldsymbol{x})-C_{i j k l}^{0}\right]^{2}\right\rangle}{\left(C_{i j k l}^{0}\right)^{2}}
$$

where $i j k l$ has the proper value for the longitudinal wave or shear wave. For example, for longitudinal and shear waves propagating along the $\boldsymbol{e}_{2}$ direction within the untextured polycrystals with cubic symmetry, it can be respectively approximated as:

$$
\epsilon_{L}^{2} \cong\left(\frac{4}{525}\right)\left[\frac{\left(a_{c}\right)^{2}}{\left(C_{2222}^{0}\right)^{2}}\right], \epsilon_{S}^{2} \cong\left(\frac{3}{700}\right)\left[\frac{\left(a_{c}\right)^{2}}{\left(C_{1212}^{0}\right)^{2}}\right]
$$

This fundamental assumption makes sure that the variation of elastic properties from crystallite to crystallite is small. Furthermore, the formula of the backscattering coefficient is expected to be valid for the early time portion so that the scattering-induced attenuation is not significant [7].

Finally 3D and 2D analytical curves of longitudinal-to-longitudinal backscattering coefficient versus frequency $f \in[0,16] \mathrm{MHz}$ for the polycrystalline material studied in this work are presented for three grain sizes of $80 \mu \mathrm{m}, 160 \mu \mathrm{m}$ and $320 \mu \mathrm{m}$ (Fig. 3.(a)). The material properties of the studied material, a titanium alloy [26], are presented in Table 1. Its anisotropy degree for longitudinal waves is twice larger than the one of weak-scattering material of aluminum [2].

\begin{tabular}{|c|c|c|c|c|c|c|}
\hline \multirow{2}{*}{ Properties } & \multicolumn{3}{|c|}{ Elastic Stiffness Constant } & Relative & Relative & \\
\cline { 2 - 4 } & $C_{1111}(\mathrm{GPa})$ & $C_{1122}(\mathrm{GPa})$ & $C_{2323}(\mathrm{GPa})$ & Anisotropy $\epsilon_{L}$ & Anisotropy $\epsilon_{S}$ & $\rho\left(\mathrm{kg} / \mathrm{m}^{3}\right)$ \\
\hline $\begin{array}{c}\text { Heterogeneous } \\
\text { polycrystalline material }\end{array}$ & 134.0 & 110.0 & 36.0 & $2.74 \times 10^{-2}$ & $8.73 \times 10^{-2}$ & 4428.0 \\
\hline $\begin{array}{c}\text { Reference homogeneous } \\
\text { and isotropic material }\end{array}$ & 153.0 & 100.0 & 26.5 & 0.0 & 0.0 & 4428.0 \\
\hline
\end{tabular}

Table 1. Material Properties of the studied polycrystalline material

Fig. 3.(a) shows that the larger grains backscatter more energy at low frequency, whereas at high frequency, smaller grains backscatter more energy and the backscattering coefficient of the largest grain becomes constant. The scattering plateau can be attributed to the transition from the stochastic scattering region to the geometric one. Otherwise the comparison between the $3 \mathrm{D}$ and the $2 \mathrm{D}$ cases shows that the $2 \mathrm{D}$ case gives rise to larger backscattering coefficients than the $3 \mathrm{D}$ case as predicted by the effect of the multiplier coefficient $\pi \sqrt{\left(x_{0}\right)^{-2}+1}$ and that the asymptotic value in high frequency range for the $2 \mathrm{D}$ case is $\pi$ times the one for the $3 \mathrm{D}$ case. Finally as referred to (42) and (43), the differential backscattering cross section per crystallite $\eta d$ is independent of the grain size and a master curve is therefore obtained (Fig. 3.(b)). 
(a)

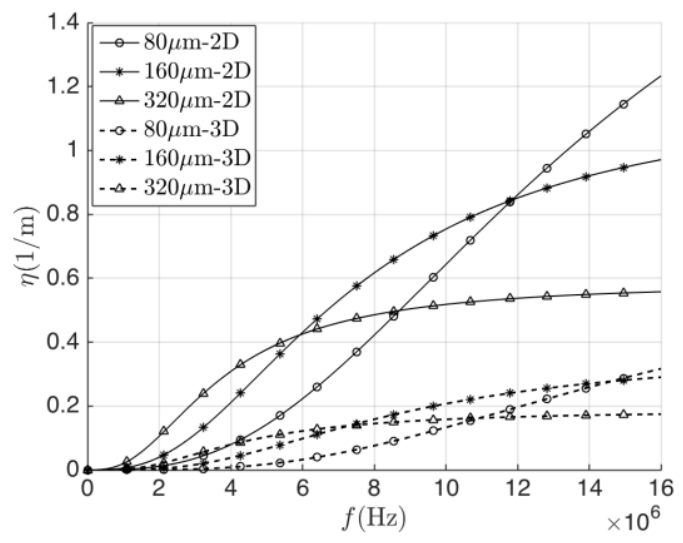

(b)

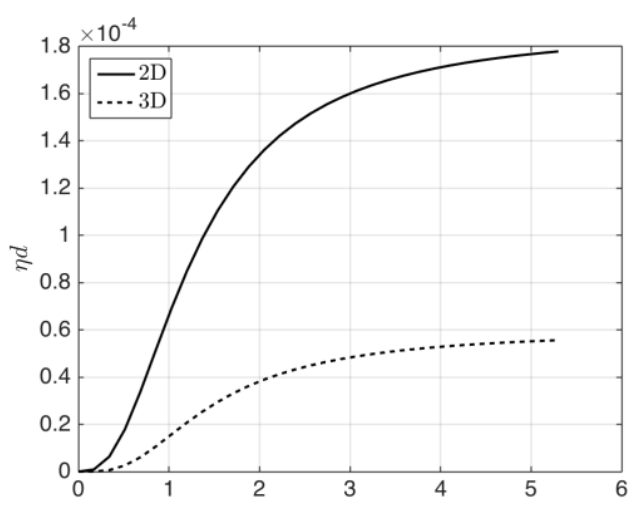

Fig. 3. Analytical results of longitudinal-to-longitudinal backscattering coefficient for different grain sizes in the 3D and 2D cases (a) $\eta$ as function of $f$; (b) master curves $\eta d$ as function of $x_{0}$

\subsection{Numerical measure of the scattering coefficient}

Within the theoretical framework based on Auld's reciprocity theorem, we define a new numerical approach for the evaluation of the microstructural noise scattering coefficient using FE simulations.

We propose to utilize the same procedure shown in Fig. 1.: Considering a single-phase, macroscopically isotropic and homogeneous polycrystalline medium with randomly oriented grains, for a given distribution of grain crystallographic orientations denoted by $\Theta$, two FE transient simulations are performed in the time domain. The first one in the heterogeneous media of interest, by applying external loadings (prescribed displacements or surface forces) on the emitter part of boundary $\Sigma_{1}$, and by recording displacements on the receiver part of boundary $\Sigma_{2}$. The second one is performed with the homogeneous reference medium by interchanging roles played by $\Sigma_{1}$ and $\Sigma_{2}$. The rest of the boundary $\Sigma$ remains free surface. With such a configuration, the unit vector directed from $\Sigma_{1}$ toward $\Sigma_{2}$ defines the scattering direction $\boldsymbol{e}_{\text {scat }}$ of interest. When $\Sigma_{1}$ coincides with $\Sigma_{2}$, the backscattering is considered.

As the scattered solution $\boldsymbol{u}^{1 \rightarrow 2}$ is completely given by the FE analysis for a given distribution $\Theta$, there is no need to use the Born approximation. According to (38), the Fourier transform in the frequency domain of FE data are then used to calculate the scattering coefficient as follows:

$$
\eta\left(\omega, \boldsymbol{e}_{\text {scat }}, \Theta\right)=\frac{1}{V_{p c}} \frac{1}{\left(U_{L}\right)^{2}\left(U_{L}^{0}\right)^{2}} \frac{\left|\chi\left(k_{0 L}, N_{\text {dim }}\right)\right|^{2}}{\left(4 \pi \rho^{0} v_{0 L}^{2}\right)^{2}}\left(\frac{4 P_{0}(\omega)}{\omega}\right)^{2} \delta S(\omega, \Theta) \delta S^{*}(\omega, \Theta)
$$

where $U_{L}$ and $U_{L}^{0}$ are respectively the averaged displacement amplitudes of incident waves in both simulations. The amplitude of scattered signals $\delta S(\omega, \Theta)$ is calculated as integration over the boundary instead of the volume for the sake of numerical effectiveness:

$$
\delta S(\omega, \Theta)=\frac{-i \omega}{4 P_{0}(\omega)}\left(\left(\boldsymbol{T}, \boldsymbol{u}^{0 ; 2 \rightarrow 1}(\boldsymbol{x})\right)_{\Sigma_{1}}-\left(\boldsymbol{T}^{0}, \boldsymbol{u}^{1 \rightarrow 2}(\boldsymbol{x})\right)_{\Sigma_{2}}\right)
$$


with the stress vectors $\boldsymbol{T}=\boldsymbol{\sigma}^{1 \rightarrow 2} \cdot \boldsymbol{n}$ on $\Sigma_{1}$ and $\boldsymbol{T}^{0}=\boldsymbol{\sigma}^{0 ; 2 \rightarrow 1} \cdot \boldsymbol{n}$ on $\Sigma_{2}$ and the displacements $\boldsymbol{u}^{1 \rightarrow 2}$ on $\Sigma_{2}$ and $\boldsymbol{u}^{0 ; 2 \rightarrow 1}$ on $\Sigma_{1}$ given by both simulations.

Finally by performing FE simulations on the samples with different distributions $\Theta$, the scattering coefficient of the polycrystalline medium is estimated in the following way:

$$
\eta\left(\omega, \boldsymbol{e}_{\text {scat }}\right)=\left\langle\eta\left(\omega, \boldsymbol{e}_{\text {scat }}, \Theta\right)\right\rangle
$$

For real experimental or numerical tests in general cases, it is tricky to estimate $V_{p c}$ the volume of grains involved in the scattering. We propose here to define two limits of $V_{p c}$ :

The lower limit $V_{\text {pcMin }}$ is the volume of the "straight beam" connecting the two surfaces $\Sigma_{1}$ and $\Sigma_{2}$ and it can be shown that a good estimate of $V_{p c M i n}$ reads as:

$$
V_{p c M i n} \approx \frac{1}{2}\left(\left|\Sigma_{1}\right|+\left|\Sigma_{2}\right|\right) \min \left(v_{0 L} t_{f}, d_{\Sigma_{1} \Sigma_{2}}\right)
$$

with $\left|\Sigma_{m}\right|$ the area of $\Sigma_{m}(m=1,2), t_{f}$ the total observation time and $d_{\Sigma_{1} \Sigma_{2}}$ the distance between the centers of $\Sigma_{1}$ and $\Sigma_{2}$.

The upper limit $V_{\text {pcMax }}$ is the volume of all grains that can be traversed by waves propagating from $\Sigma_{1}$ to $\Sigma_{2}$ for $t \leq t_{f}$, not only the grains lying on the straight paths connecting $\Sigma_{1}$ and $\Sigma_{2}$ considered by the lower limit. In the case where the geometry of $\Omega$ is convex, it can be shown that a good estimate of $V_{p c M a x}$ is:

$$
V_{\text {pcMax }} \approx V_{\text {pcMin }}+\operatorname{Vol}\left(\Omega \cap \operatorname{Ellip}\left(\Sigma_{1}, \Sigma_{2}, t_{f}\right)\right)
$$

where Ellip $\left(\Sigma_{1}, \Sigma_{2}, t_{f}\right)$ denotes an ellipsoid with two equal semi-diameters and with the centers of $\Sigma_{1}$ and $\Sigma_{2}$ as the two foci on the third principal axis, whose length is equal to $v_{0 L} t_{f}$. In the particular case of the measurement of the backscattering coefficient, $\Sigma_{1}$ and $\Sigma_{2}$ are completely superposed, the calculation of $V_{\text {pcMin }}$ does not change, while for $V_{p c M a x}, \operatorname{Ellip}\left(\Sigma_{1}, \Sigma_{2}, t_{f}\right)$ becomes a sphere with the center of $\Sigma_{1}$ as its center and $v_{0 L} t_{f}$ as diameter.

Then to compare numerical calculation and the theoretical estimation of the scattering coefficient, we propose to compare $\eta V_{p c}$ instead of $\eta$. That means the following numerical output:

$$
\left(\eta\left(\omega, \boldsymbol{e}_{s c a t}\right) V_{p c}\right)_{n u m}=\frac{1}{\left(U_{L}\right)^{2}\left(U_{L}^{0}\right)^{2}} \frac{\left|\chi\left(k_{0 L}, N_{d i m}\right)\right|^{2}}{\left(4 \pi \rho^{0} v_{0 L}^{2}\right)^{2}}\left(\frac{4 P_{0}(\omega)}{\omega}\right)^{2}\left\langle\delta S(\omega) \delta S^{*}(\omega)\right\rangle
$$

is compared with the two theoretical lower and upper limits $\eta\left(\omega, \boldsymbol{e}_{s c a t}\right) V_{p c M i n}$ and $\eta\left(\omega, \boldsymbol{e}_{s c a t}\right) V_{p c M a x}$. The analytical formula of $\eta\left(\omega, \boldsymbol{e}_{\text {scat }}\right)$ is given in Section 2.5.

It is important to note that the proposed numerical procedure measures the actual reciprocity gap, as the Born approximation is not used. Multiple scattering effects, if exist, are contained within the numerically calculated scattering coefficient. Therefore our numerical testing procedure breaks through the limitations of the use of the Born approximation and the single-scattering assumption made in the theoretical evaluation. It has the ability to simulate precisely the time-domain noise 
signals of elastic wave propagating in polycrystalline materials including complex physical mechanisms, such as multiple scattering and attenuation. Thus it has wider generality and higher accuracy than the analytical evaluation and can be applicable to complex media with strong scattering due to high degree of anisotropy, textured microstructure or multi-phase microstructure etc. Furthermore, the effectiveness of our procedure based on reciprocity theorem is obvious, since it is much easier to record and integrate displacement and stress fields at the boundary rather than in the volume. This approach is versatile since the scattering coefficient in any given direction can be evaluated by only changing the position of the receivers.

\section{FE modeling of elastic wave propagation in polycrystalline materials}

FE simulations of ultrasonic wave propagation in polycrystalline materials presented in this work are performed by using an in-house software OOFE (Object Oriented Finite Element program) [14, 27]. The elastodynamic equations governing elastic wave propagation are solved in the time domain by an implicit solver based on a time-discontinuous space-time Galerkin method [17, 27]. Grain size effects on the backscattering coefficient of single-phase and equiaxed polycrystalline materials with randomly distributed crystallographic orientations are numerically investigated. According to the theoretical analysis, the equivalent homogeneous and isotropic medium defined by the Voigt average of the anisotropic elastic stiffness tensor over all crystallographic orientations is taken as the reference medium. The Voigt average stiffness tensor is given in Tables 1 and 2. Only 2D FE simulations are presented in the present work.

\subsection{Definition of 2D FE models of ultrasonic wave propagation}

2D FE models used in the present work to calculate the backscattering coefficient are shown in Fig. 4.. The first one considers the polycrystalline material of interest (the grey part in Fig. 4.(a)) embedded in the homogeneous reference material (the white part in Fig. 4.(a)). The whole domain is chosen sufficiently large in order to avoid arrival on the receiver of waves reflected by the external lateral boundaries during the time interval of analysis. The second one (Fig. 4.(b)) considers the reference material. The same FE mesh is used to discretize both heterogeneous and homogeneous reference domains.

The studied polycrystalline materials have an idealized theoretical microstructure with hexagonal grains. It is single phase with cubic crystal symmetry. Crystallographic orientations of grains defined in terms of the Euler angles $\Theta\left(\varphi_{1}, \phi, \varphi_{2}\right)$, which define the anisotropic axes with respect to the global Cartesian basis, are randomly distributed by providing the following set up:

$$
\begin{gathered}
\varphi_{1}=\text { random }[0,2 \pi[ \\
\phi=\operatorname{acos}(\operatorname{random}[-1,1]) \\
\varphi_{2}=\operatorname{random}[0,2 \pi[
\end{gathered}
$$


(a)

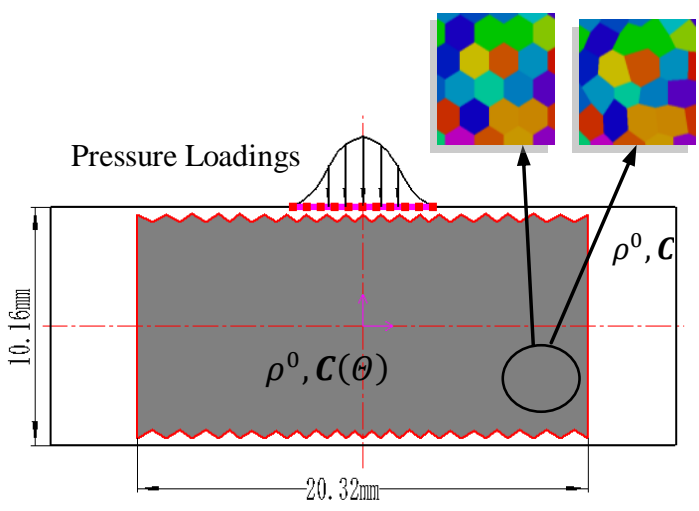

(b)

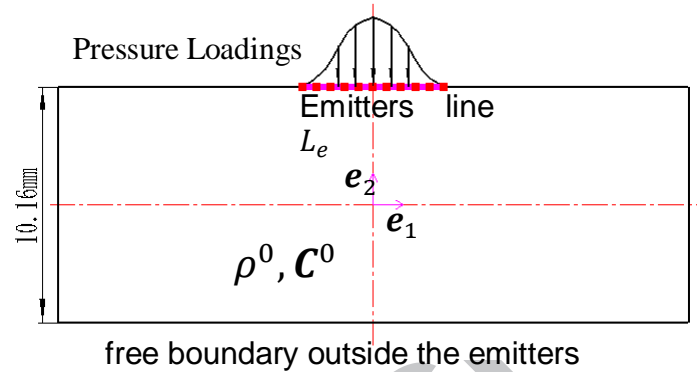

Fig. 4. 2D FE models for (a) the heterogeneous polycrystalline medium; (b) the homogeneous reference medium.

In this work, regular hexagonal grains are mainly considered. For a regular hexagonal grain, its grain size is defined as the diameter of its inscribed circle and denoted by $H$. The equivalent grain size defined as the diameter of the circular grain having the same grain surface is then $d=1.05 \mathrm{H}$. FE models for the samples with single-size regular hexagonal grains, with three different grains sizes $H=80 \mu \mathrm{m}, 160 \mu \mathrm{m}$ and $320 \mu \mathrm{m}$, are defined. For the three considered grain sizes, the polycrystalline domain is composed of respectively 35630,8855 and 2125 randomly oriented hexagonal crystallites. For a given grain size, ten different random distributions of crystallographic orientations are considered.

Furthermore, to investigate if there are any numerical artifacts due to the use of the regular hexagonal grains, which results in regularly oriented grain boundaries (Fig. 4.(a)), two microstructures composed of geometrically varying grains are considered: an irregular hexagonal grain morphology obtained by slight and random perturbation of regular hexagonal grain shape, and a random grain morphology generated by using the Voronoi approach. Ten samples of different crystallographic orientations for each case are carried out. The simulation with irregular hexagonal grain shape has no other goal than proving the specific orientations of the grain boundaries do not matter. Accordingly we keep the same center points and the same crystallographic orientation for each grain, but changed only the grain boundary orientations. By contrast, the crystallographic orientation distributions for the ten samples with a Voronoi-type grain morphology can be more or less different as grains are randomly shaped.

Equivalent grain sizes $(H, d)$ are calculated for each grain using the hexagonal regular grain having the same grain surface. For the samples with irregular grains, Fig. 5.(a) and (b) show in blue the distribution of angles formed by grain boundaries with the horizontal axis and the distribution of grain size, respectively. Both distributions for samples composed of regular grains are also shown with red vertical lines. Concerning the grain boundary orientation, it is shown that the distribution of the orientations of irregular hexagonal grain boundaries is the sum of three Gaussian distributions with mean value respectively equal to the three orientations $-90^{\circ},-30^{\circ}$ and $30^{\circ}$ of regular grain boundaries. 
Concerning the distribution of grain size, it reveals that microstructures with irregular hexagonal grains present a Gaussian grain size distribution with the unweighted average equal to the size of regular grains. By contrast, for the Voronoi tessellation, a random distribution of seeds leads to a random distribution of grain boundary orientation (Fig. 5.(c)), and a Gaussian grain size distribution is also generated with the same average grain size and slightly larger standard deviation than the one of irregular hexagonal grains. Numerical results on these samples allow ruling out any suspicion of possible artifact due to the use of regular hexagonal grains for the numerical simulations (Section 4.2).

(a)

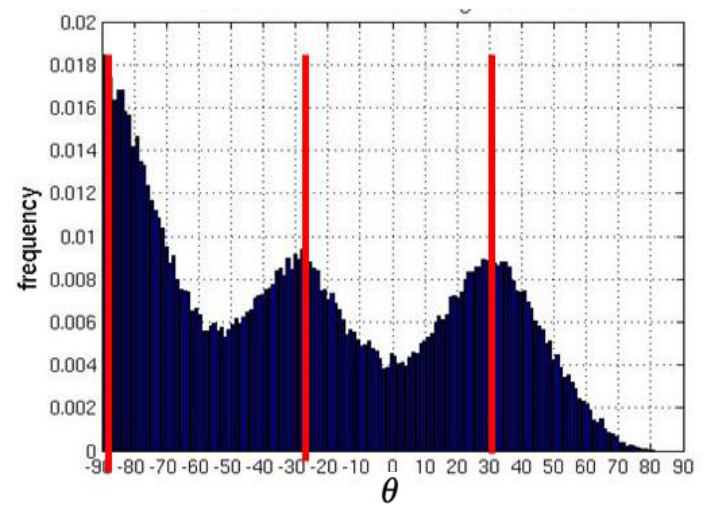

(c)

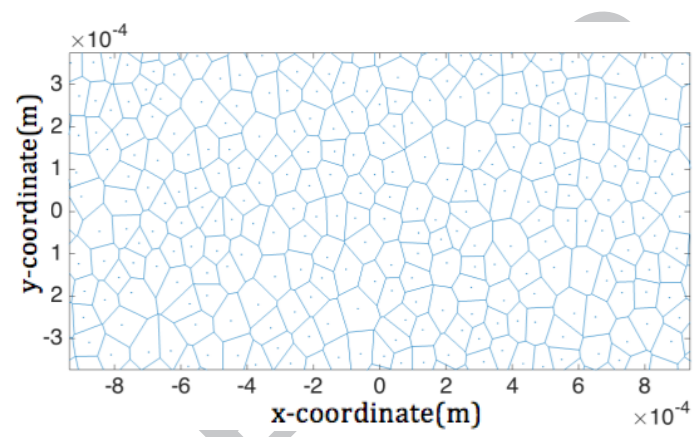

(b)
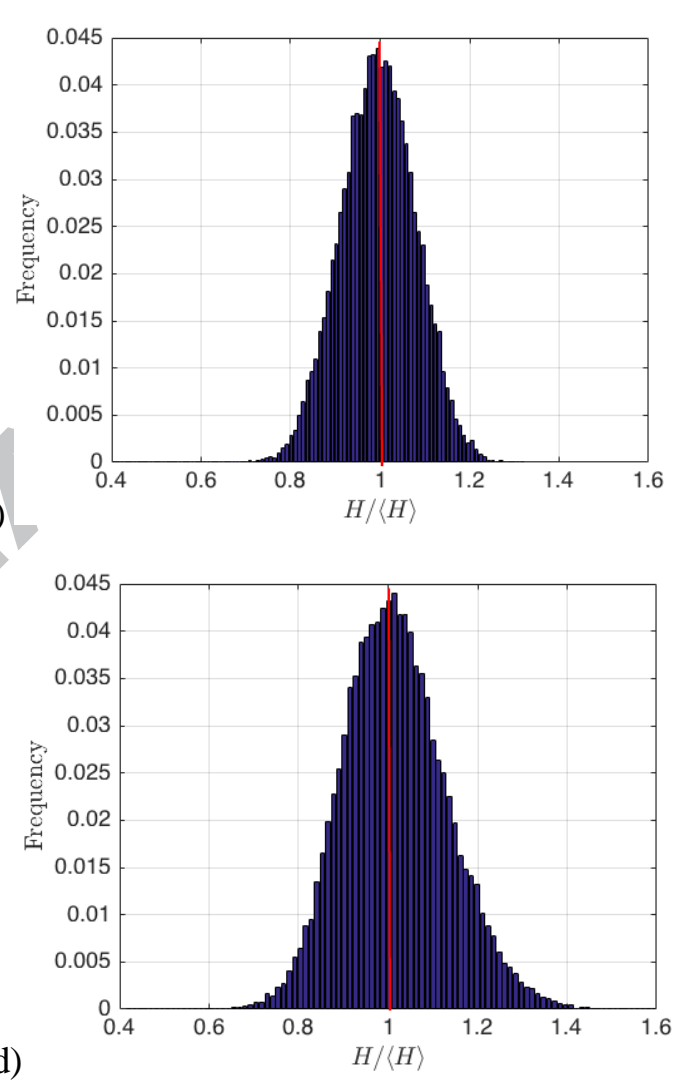

(d)

Fig. 5. (a) Grain boundary orientation distributions w.r.t. the horizontal axis and (b) grain size distributions for polycrystals with irregular hexagonal grains; (c) Voronoi-type grain morphology and (d) its grain size distribution. In blue: Gaussian type distributions for microstructures with geometrically varying grains with $\langle H\rangle=80 \mu \mathrm{m}$; In red: Vertical lines for microstructures with regular hexagonal grains with $H=80 \mu m$

Concerning the external loadings and the data recording, an emitter line $L_{e}$ of $2.8 \mathrm{~mm}$ length on the external top boundary of the studied domain is defined (Fig. 4). Twenty-two uniformly distributed points are defined on $L_{e}$ and serve as emitters and receivers. To simulate a broadband ultrasonic incident signal produced by a laser pulse [23], ultrasonic waves are generated by applying a pressure loading $p(x, t) \boldsymbol{e}_{2}$ on $L_{e}$. For a given $x, p(x, t)$ is the sum of two Ricker signals varying in time within a period $T_{r}$, whose central peak frequency $f_{c}=2 / T_{r}$ is respectively equal to $5 \mathrm{MHz}$ and $10 \mathrm{MHz}$. Each Ricker signal ricker $(x, t)$ reads as: 
(a)

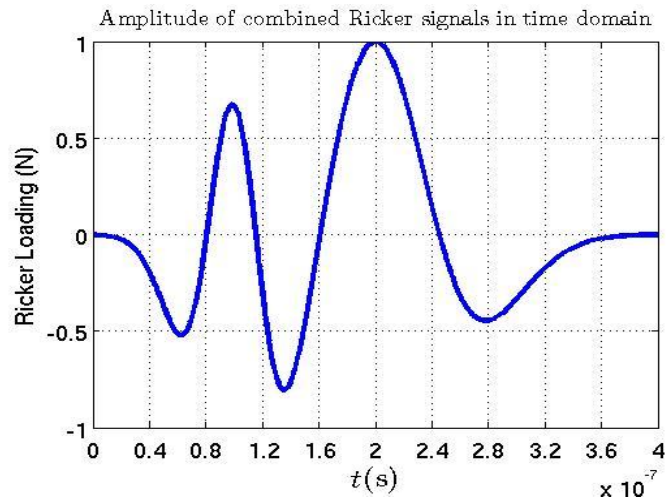

(b)

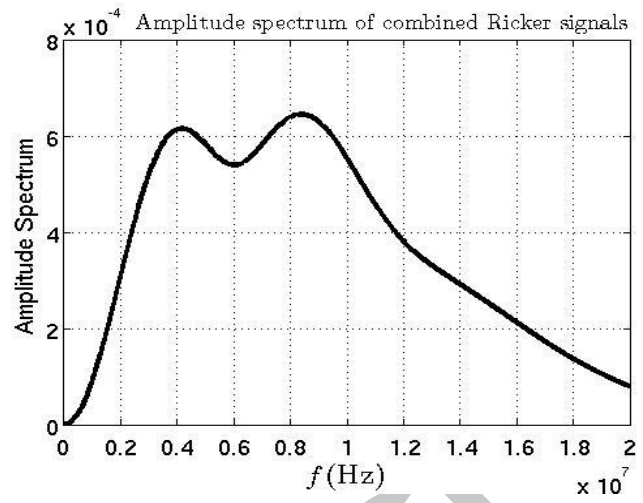

Fig. 6. Combined Ricker signal used as the external pressure loadings presented in both (a) time and (b) frequency domains

$$
\operatorname{ricker}(x, t)=\left\{\begin{array}{cc}
A_{r}(x)\left(1-2 \pi^{2} f_{c}^{2}\left(t-\frac{1}{f_{c}}\right)^{2}\right) \exp \left(-\pi^{2} f_{c}^{2}\left(t-\frac{1}{f_{c}}\right)^{2}\right), & \text { for } 0 \leq t \leq 2 / f_{c} \\
0, & \text { for } t \geq 2 / f_{c}
\end{array}\right.
$$

Furthermore in order to reduce effects of shear waves, which would be triggered when discontinuities in boundary conditions occur at the ends of the emitter line, the peak amplitudes $A_{r}(x)$ of both Ricker signals are chosen to have a Gaussian distribution along the emitter line. Thus the peak amplitudes of input signals smoothly decrease down to zero at the ends of the emitter, which is better suited to the free boundary condition imposed on the other side. Fig. 6. presents the external pressure loading used in the present work in both time and frequency domains. The quality of FE simulations can be deteriorated in frequency ranges where the spectral amplitude of incident waves are very small, therefore according to the frequency spectrum of the combined Ricker signal (Fig. 6.(b)), the valid frequency domain of our numerical simulations presented herein is about $[2,16] \mathrm{MHz}$.

Unstructured FE meshes using both triangular and quadrilateral finite elements have been used in the numerical simulations without significant differences. Hence only simulations using quadrilateral elements are presented here. An example of FE mesh of microstructures with the grain size $H=$ $320 \mu \mathrm{m}$ is presented in Fig. 7 and shows the great flexibility offered by the FEM to exactly models the grain boundaries.

(a)

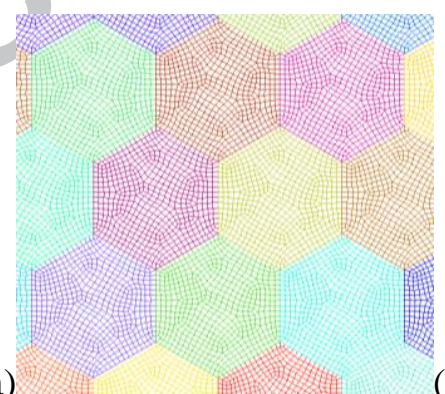

(b)

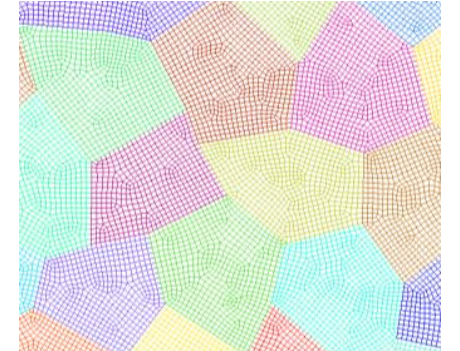

(c)

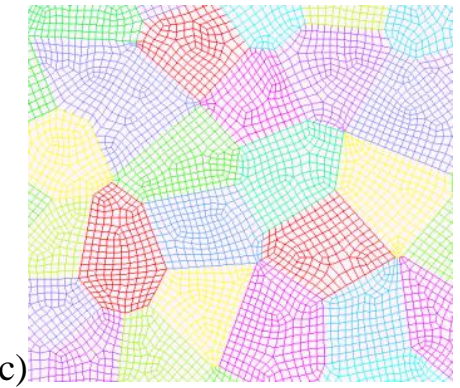

Fig. 7. FE meshes of microstructures composed of (a) regular and (b) irregular hexagonal grains and (c) a random Voronoi-type grain morphology with averaged grain size of $320 \mu \mathrm{m}$. 


\subsection{Mesh convergence analysis}

In order to account for the impedance variations between two adjacent grains and have a good representation of wave front, a sufficient number of elements included in one grain and in the shortest wavelength of interest is essential for the quality of numerical simulations [16, 27]. However, the computational cost increases greatly when the mesh becomes more refined. To control the quality and optimize the efficiency, the mesh convergence analysis is of great importance.

For the mesh convergence analysis presented in this Section, FE models with all the three grain sizes $80 \mu \mathrm{m}, 160 \mu \mathrm{m}$ and $320 \mu \mathrm{m}$ are used but they are only of half sizes of those described in Fig. 4. in order to reduce the computational cost. Different ratios of grain size to element size $d / h$ are considered. The following mechanical quantity $\zeta(f)$ is chosen to quantify the mesh convergence:

$$
\zeta(f)=\frac{\sqrt{\frac{1}{M} \sum_{j=1}^{j=M}\left(v_{2}^{b}\left(\boldsymbol{x}_{j}, f\right)\right)^{2}}}{\sqrt{\frac{1}{M} \sum_{j=1}^{j=M}\left(v_{02}\left(\boldsymbol{x}_{j}, f\right)\right)^{2}}}
$$

where $M=22$ is the total number of emitter/receiver probes, $v_{2}^{b}\left(\boldsymbol{x}_{j}, f\right)$ and $v_{02}\left(\boldsymbol{x}_{j}, f\right)$ represent respectively the backscattered noise and the incident signals, they are in fact the Fourier transform of the corresponding velocity component in the direction $\boldsymbol{e}_{2}$ recorded at the jth probe $\boldsymbol{x}_{j} \cdot \zeta(f)$ is therefore a frequency-dependent coefficient calculated as the ratio of the root-mean-square (rms) velocity between the backscattered noise and the incident signals. It is associated to the percentage of the energy loss due to scattering in the backscattered direction. The backscattering mesh convergence is evaluated by $\left(\zeta-\zeta_{c}\right) / \zeta_{c}$ with the converged solution $\zeta_{c}$ obtained by the highest available density mesh of all studied FE models.

Fig. 8. presents the backscattering mesh convergence for the frequency $f=10 \mathrm{MHz}$ as respectively a function of the number of elements per grain size $d / h$ and a function of the number of elements per wavelength $\lambda / h$.

On the one hand, it is seen that a sample with a smaller grain size converges more rapidly with respect to the number of elements per grain size $d / h$ (Fig. 8.(a)). Indeed for a given ratio $d / h$, a larger number of elements per wavelength $\lambda / h$ is obtained for the sample with a smaller grain size and thus results in less mesh scattering. At $f=10 \mathrm{MHz}$ and for $d / h=10$, only the grain size of $80 \mu \mathrm{m}$ has converged for the threshold of $1 \%$, which agrees with the results obtained by Van Pamel et al. [15] and Shahjahan et al. [16]. Otherwise it is worth to remark that the errors for the grain size of $320 \mu \mathrm{m}$ are significantly higher than the other two grain sizes since this grain size is comparable to the studied wavelengths.

On the other hand, a sample with a smaller grain size converge less rapidly with respect to the number of elements per wavelength $\lambda / h$ (Fig. 8.(b)), because there are less elements per grain size for a given ratio $\lambda / h$. Our analysis concludes that there should be at least 20 elements per wavelength to ensure the beginning of a converging process. 

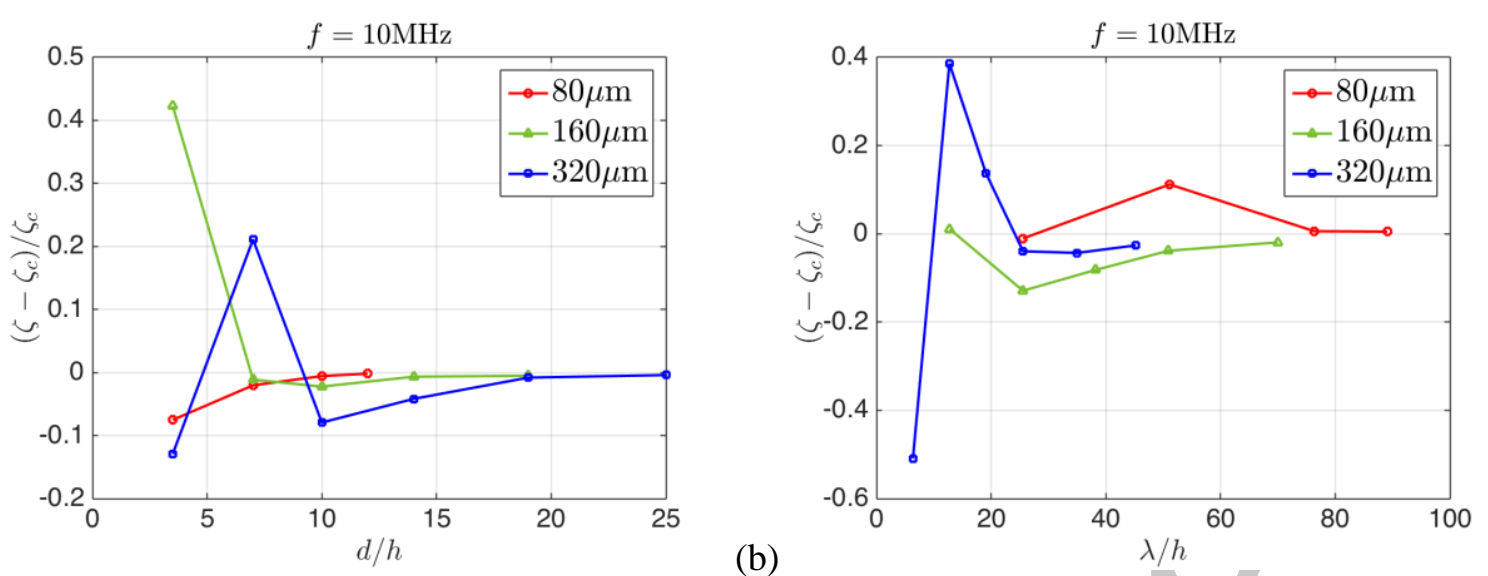

(a)

Fig. 8. Backscattering mesh convergence $\left(\zeta-\zeta_{c}\right) / \zeta_{c}$ w.r.t. (a) the number of elements per grain size $d / h$ and (b) the number of elements per wavelength $\lambda / h$ for three grain sizes for $f=10 \mathrm{MHz}$.

Fig. 9. presents the backscattering mesh convergence as a function of the number of elements per grain size $d / h$ at six different frequencies for each of the three grain sizes. For a given ratio of $d / h$, it is observed that the lower the frequency is, the more rapid the convergence is because of a larger number of elements per wavelength $\lambda / h$.

The numerical results presented in the next section are obtained using the FE meshes with $d / h=7,14$ and 25 respectively for the three grain sizes $H=80 \mu \mathrm{m}, 160 \mu \mathrm{m}$ and $320 \mu \mathrm{m}$. Therefore according to our mesh convergence analysis, the backscattering coefficient is converged at least for the threshold of $7 \%$ at all frequencies and for all three grain sizes (Fig. 9.). It can be concluded that the FE models used herein are appropriately defined and adequate convergence is achieved in the measurement of noise levels.

\subsection{Ultrasonic phase velocity in polycrystals}

In this section, different ways to calculate effective elastic properties and longitudinal wave phase velocities of the studied polycrystalline material are considered. Numerically estimated phase velocity is compared with the theoretical effective phase velocities. It allows determining which effective elastic tensor should be used as equivalent reference medium on the one hand, and on the other hand validating our FE models in the term of quality of estimating the arrival time of waves.

Firstly three averages of elastic stiffness tensor classically defined by static homogenization studies to get effective elastic properties are considered. The Voigt average results in an upper bound of effective elastic stiffness tensors under the assumption of uniform strain, i.e. $\langle\boldsymbol{C}(\boldsymbol{x}): \boldsymbol{\varepsilon}(\boldsymbol{x})\rangle=$ $\langle\boldsymbol{C}(\boldsymbol{x})\rangle: \boldsymbol{\varepsilon}^{0}$, so $\langle\boldsymbol{C}(\boldsymbol{x})\rangle^{\text {Voigt }}=\langle\boldsymbol{C}(\boldsymbol{x})\rangle$. The Reuss average results in a lower bound by assuming a uniform stress state, i.e. $\left\langle\boldsymbol{C}^{-1}(\boldsymbol{x}): \boldsymbol{\sigma}(\boldsymbol{x})\right\rangle=\left\langle\boldsymbol{C}^{-1}(\boldsymbol{x})\right\rangle: \boldsymbol{\sigma}^{0}$, so $\langle\boldsymbol{C}(\boldsymbol{x})\rangle^{\text {Reuss }}=\left\langle\boldsymbol{C}^{-1}(\boldsymbol{x})\right\rangle^{-1}$. A selfconsistent (SC) average [29] gives an estimate between the Voigt and Reuss bounds, by calculating equivalent elastic properties when either the elastic stiffness tensor $\boldsymbol{C}(\boldsymbol{x})$ or the compliance tensor $\boldsymbol{C}(\boldsymbol{x})^{-1}$ is averaged over all possible orientations. 

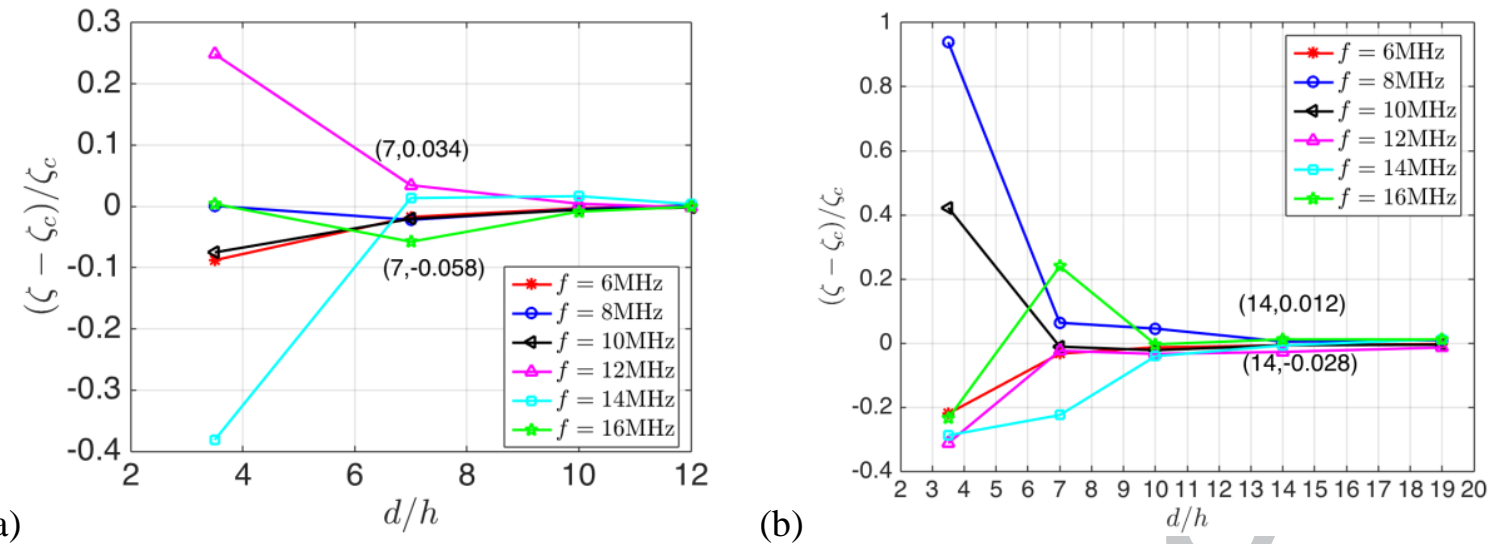

(a)

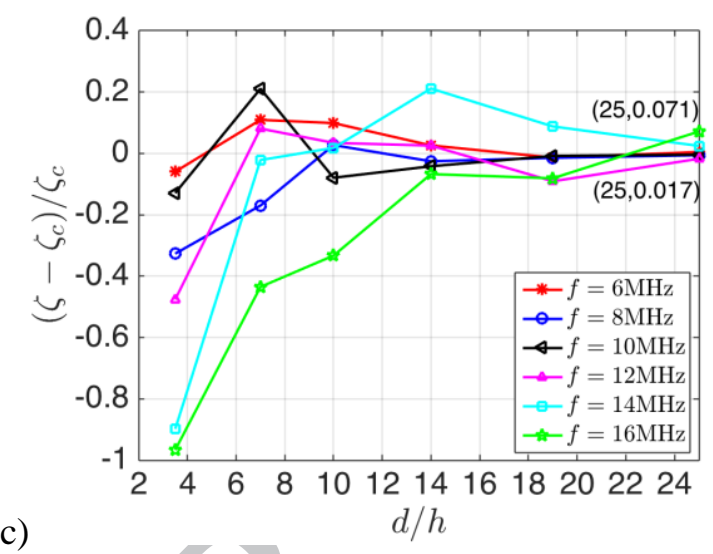

Fig. 9. Backscattering mesh convergence $\left(\zeta-\zeta_{c}\right) / \zeta_{c}$ w.r.t. the number of elements per grain size $d / h$ at six different frequencies for the grain sizes of (a) $80 \mu \mathrm{m}$, (b) $160 \mu \mathrm{m}$ and (c) $320 \mu \mathrm{m}$; The number of elements per grain size $d / h$ of the FE meshes used for the numerical analysis presented in the Section 4 and their precision are indicated for each grain size.

For a polycrystalline sample containing 2125 grains of the grain size $H=320 \mu \mathrm{m}$, the effective elastic properties of the three different averages and the corresponding phase velocities $v_{0 L}=$ $\sqrt{\left\langle C_{2222}\right\rangle / \rho^{0}}$ of longitudinal waves propagating in the direction $\boldsymbol{e}_{2}$ are listed in Table 2 . The phase velocities are quite different: Those given by the SC and Reuss averages are about $1.3 \%$ and $2.8 \%$ smaller than the one given by the Voigt average.

\begin{tabular}{|c|c|c|c|c|c|}
\hline \multirow{2}{*}{ Properties } & \multirow{2}{*}{$v_{0 L}(\mathrm{~m} / \mathrm{s})$} & \multicolumn{3}{|c|}{ Elastic Constants } \\
\cline { 4 - 7 } & & $\left\langle C_{2222}\right\rangle(\mathrm{GPa})$ & $\left\langle C_{1122}\right\rangle(\mathrm{GPa})$ & $\left\langle C_{2323}\right\rangle(\mathrm{GPa})$ \\
\hline \multirow{3}{*}{$v_{0 L}=\sqrt{\left\langle C_{2222}\right\rangle^{\mathrm{av}} / \rho^{0}}$} & $\mathrm{av}=\mathrm{Voigt}$ & 5878 & 153.0 & 100.0 & 26.5 \\
\cline { 4 - 7 } & $\mathrm{av}=\mathrm{SC}$ & 5800 & 149.0 & 102.0 & 23.9 \\
\cline { 4 - 7 } & av = Reuss & 5714 & 144.6 & 104.6 & 20.0 \\
\hline
\end{tabular}

Table 2. Effective elastic properties and phase velocities of longitudinal waves of a polycrystalline sample containing 2125 grains of size $320 \mu \mathrm{m}$ 
The numerical evaluation of phase velocities is based on a spectral autocorrelation method with numerical data recorded at 11 inner probes placed $2 \mathrm{~mm}$ below the emitter-receiver line $L_{e}$. Fig. 10 . compares, for the same polycrystalline sample containing 2125 grains of size $320 \mu \mathrm{m}$, the numerical estimate and the analytical prediction of the longitudinal-wave phase velocity in terms of relative difference with the phase velocity defined by the Voigt average tensor $\langle\boldsymbol{C}(\boldsymbol{x})\rangle^{\text {Voigt }}$. The analytical prediction is calculated using the dispersion relation developed in [28] for the 2D case, which is similar to the one obtained by Stanke and Kino for the 3D case [2]. We note that the analytical predictions are obtained by using the Voigt average medium as reference medium. Good agreement is observed between both numerical and analytical predictions. This confirms the relevancy of the classical choice of using the Voigt average to determine the equivalent homogenized elastic tensor and wave velocities in polycrystalline materials with randomly oriented grains,

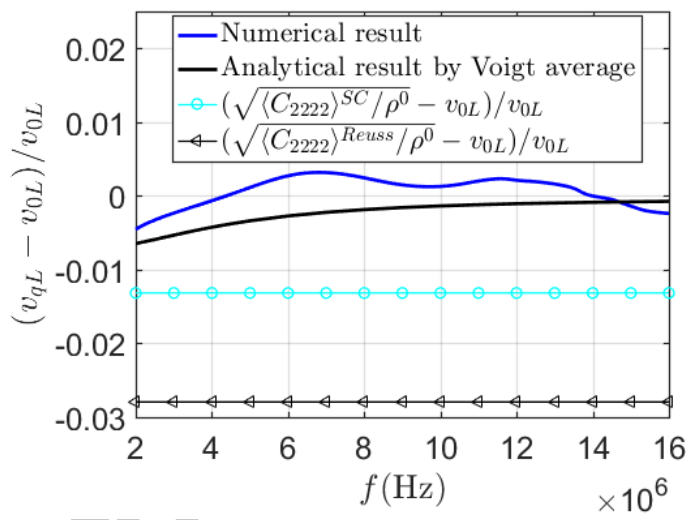

Fig. 10. Comparison between numerical and analytical estimates of the longitudinal-wave phase velocity $v_{q L}$, in a polycrystalline sample containing 2125 grains of size $320 \mu \mathrm{m}$, in terms of relative difference with the phase velocity in the Voigt average reference medium $v_{0 L}=\sqrt{\left\langle C_{2222}\right\rangle^{\text {Voigt }} / \rho^{0}}$.

\subsection{Post-processing of numerical ultrasonic data}

Two kinds of post-processing of FE data are used here.

The first one is based on the Helmholtz's decomposition: Displacement fields are decomposed into two vector fields, the one curl-free and the other divergence-free. In the case of isotropic and homogenous elastic media, the curl-free part $\boldsymbol{u}_{L}$ is in fact the longitudinal wave component as it is solution of the longitudinal wave equation, while the divergence-free part $\boldsymbol{u}_{S}$ is the shear wave component being solution of the shear wave equation [24, 30]. Therefore by displaying the scalar field $\operatorname{div} \boldsymbol{u}\left(=\operatorname{div} \boldsymbol{u}_{L}\right)$ and the vector field $\operatorname{curl} \boldsymbol{u}\left(=\operatorname{curl} \boldsymbol{u}_{S}\right)$, longitudinal and shear wavefronts are respectively represented.

Now we consider the case of 2D anisotropic and homogeneous elastic media in which a quasilongitudinal or a quasi-shear wave of wave vector $k$ propagates: $\boldsymbol{u}=\boldsymbol{U} \exp (i(\boldsymbol{k} \cdot \boldsymbol{x}-\omega t))$, with $\boldsymbol{U}=U_{k} \boldsymbol{e}_{k}+U_{k \perp} \boldsymbol{e}_{k \perp}$, where $U_{k}$ and $U_{k \perp}$ are the amplitude components respectively parallel and perpendicular to the wave vector, $\boldsymbol{e}_{k}$ and $\boldsymbol{e}_{k \perp}$ being unit vectors. Then we have: 


$$
\begin{gathered}
\operatorname{div} \boldsymbol{u}=U_{k}|\boldsymbol{k}| \exp (i(\boldsymbol{k} \cdot \boldsymbol{x}-\omega t)) \\
\text { curl } \boldsymbol{u}=U_{k \perp}|\boldsymbol{k}| \boldsymbol{e}_{k} \times \boldsymbol{e}_{k \perp} \exp (i(\boldsymbol{k} \cdot \boldsymbol{x}-\omega t))
\end{gathered}
$$

Therefore the field div $\boldsymbol{u}$ can still reveal the wavefronts of quasi-longitudinal waves since $\left\|U_{k \perp}\right\| \ll$ $\left\|U_{k}\right\|$ generally remains true, and the field curl $\boldsymbol{u}$ can still reveal the wavefronts of quasi-shear waves since $\left\|U_{k}\right\| \ll\left\|U_{k \perp}\right\|$ remains generally true. Finally in the general case of anisotropic and heterogeneous media, it is impossible to distinguish the different wave modes generated by the complex scattering at the grain boundaries behind the principle wavefronts. However, this posttreatment based on the Helmholtz's decomposition remains interesting and is used in the present work because it allows revealing the propagation of the principle wavefronts in these complex polycrystalline media.

As an example, Fig. 11. shows wavefronts of quasi-longitudinal waves and quasi-shear waves in a polycrystalline sample with the grain size $H=80 \mu \mathrm{m}$ at three different moments. We observe a principal quasi-longitudinal wavefront ( $\operatorname{div} \boldsymbol{u}$ predominant) propagating in the direction $-\boldsymbol{e}_{2}$ at first and then in the opposite direction after being reflected by the bottom of the sample. Behind the principal quasi-longitudinal wavefront, scattered noises in terms of div $\boldsymbol{u}$ are easily recognized. Fig. 11. shows also two principal quasi-shear wavefronts ( $\mid$ curl $\boldsymbol{u} \mid$ predominant). The faster one propagates together with the principal quasi-longitudinal wavefront because curl $\boldsymbol{u}$ vanish no longer on the quasilongitudinal wave fronts, but also due to the conversion from quasi-longitudinal waves to quasi-shear waves every time when the quasi-longitudinal waves encounter a grain boundary. The slower one propagates with the velocity of quasi-shear waves, nearly two times smaller than the velocity of quasilongitudinal waves. It is obvious that the quasi-shear wave modes seem to be more sensitive to grain boundaries than the quasi-longitudinal wave modes and result in high-level noises in terms of $|\mathbf{c u r l} \boldsymbol{u}|$.

The second kind of post-processing is the calculation of the backscattering coefficient according to the formula (49) and (53) developed in Section 2.4. To do this, the prescribed external pressure loadings and the velocity components $v_{2}$ in the direction $\boldsymbol{e}_{2}$ recorded at the 22 receivers on the emitter-receiver line $L_{e}$ are used. As an example, Fig. 12. illustrates the normalized velocities $\frac{v_{2}}{\max \left(v_{2}\right)}$ observed at the $10^{\text {th }}$ receiver located at the middle of $L_{e}$ for a polycrystalline sample with grain size of $80 \mu \mathrm{m}$ and for the homogeneous reference medium. The first and second peaks on both curves correspond to the incident signals and the echo signals reflected by the bottom side of the sample. For the polycrystalline sample, perturbations of much smaller amplitude than incident and echo signals are in fact backscattered noise signals, which are absent in the reference case. 

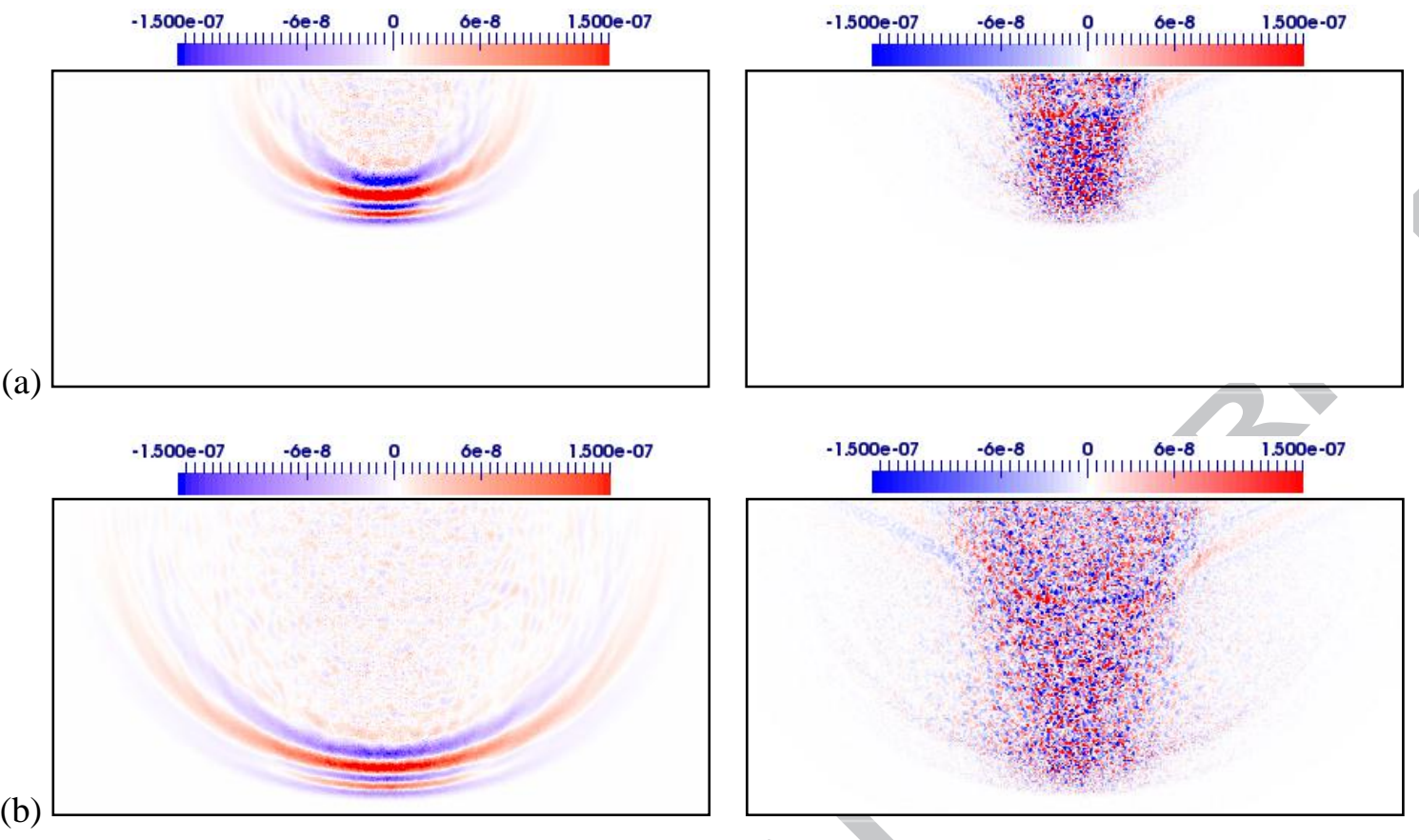

(b)
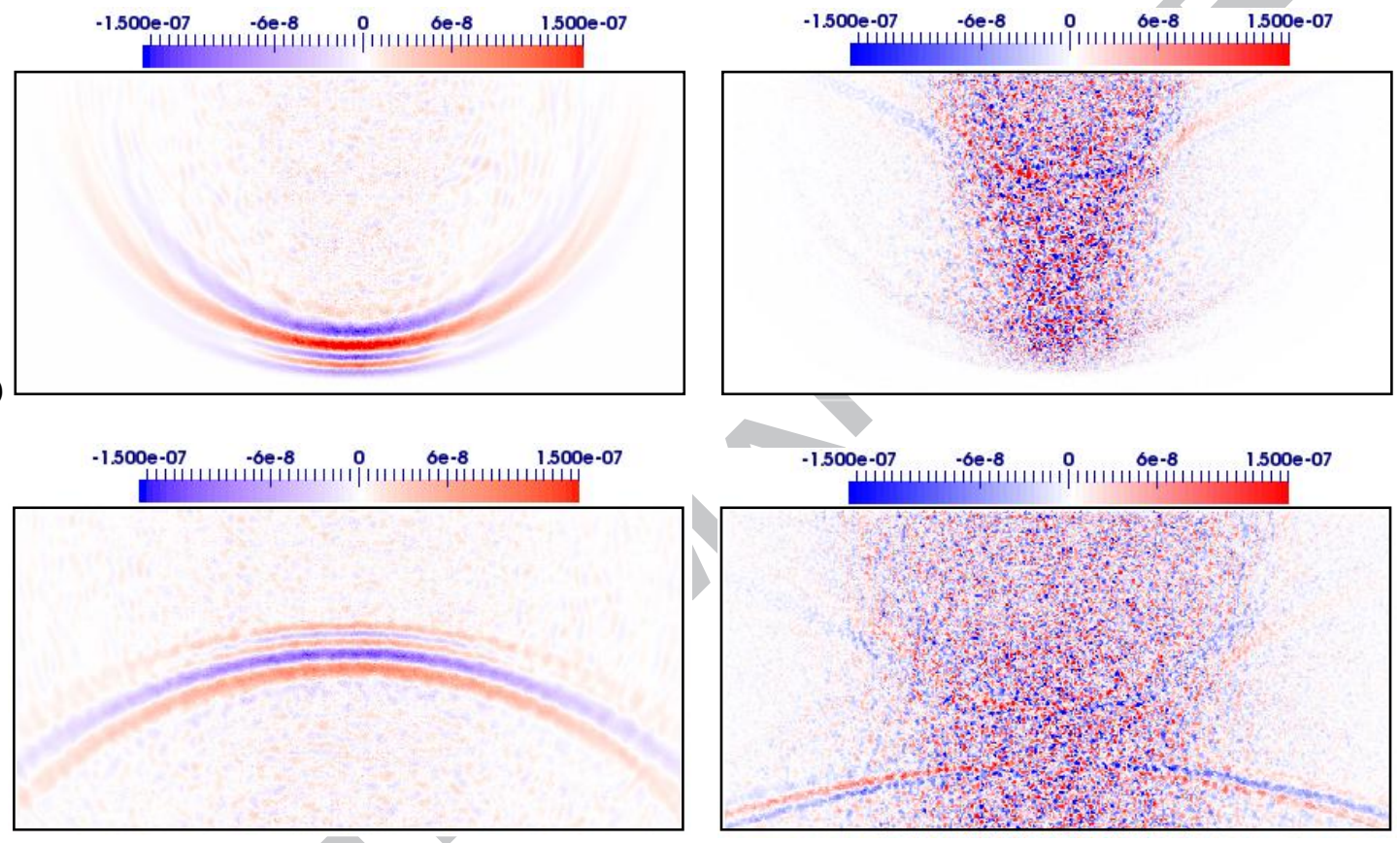

(c)

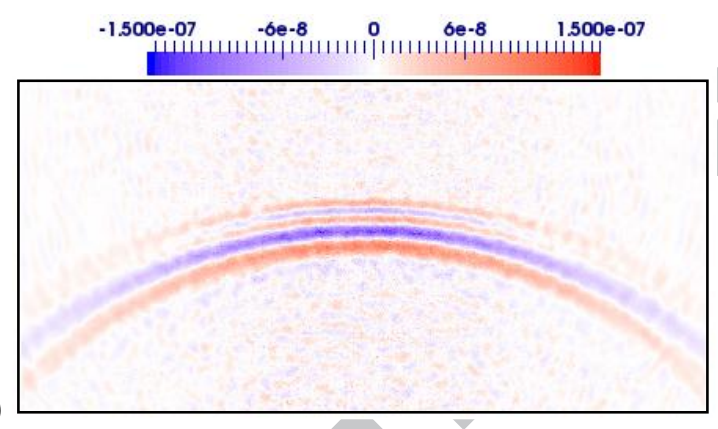

Fig. 11. Propagation of the quasi-longitudinal waves in terms of div $\boldsymbol{u}$ (left) and of the quasi-shear waves in terms of $|\operatorname{curl} \boldsymbol{u}|$ (right) in a polycrystalline sample with grain size of $80 \mu \mathrm{m}$ at different moments (a) $t=0.84 \mu \mathrm{s}$, (b) $t=1.64 \mu \mathrm{s}$ and (c) $t=2.84 \mu \mathrm{s}$.

The noise signals are recorded during a time period $\left[0, T_{f}\right]$ (Fig. 12), where $T_{f}=3.4 e-6 \mathrm{~s}$ is the arrival time of the reflected echoes at the receivers. The comparison between the theoretical and the numerical calculations requires the volume of the involved polycrystalline microstructure to be defined (Section 2.4.1). A minimum limit and a maximum limit, $V_{p c M i n}$ and $V_{p c M a x}$, are proposed in equations (51) and (52). In the $2 \mathrm{D}$ case, these are in fact surface areas, $S_{p c M i n}$ and $S_{p c M a x}$, respectively corresponding to the pink rectangular area and the green semi-circular area shown in Fig. 13.. Comparing Fig11.(b) and Fig. 13, it is interesting to point out that the minimum and maximum limits, $S_{p c \text { Min }}$ and $S_{\text {pcMax }}$, more likely correspond to the active grain volumes of respectively longitudinal-to-shear wave scattering and longitudinal-to-longitudinal wave scattering. Furthermore, the positional averaged displacement amplitudes of incident waves $U_{L}$ in (53) is calculated by: $U_{L}=1 / M \sum_{j=1}^{M}\left|U_{L}\left(x_{j}, f\right)\right|^{2}$, with $U_{L}\left(x_{j}, f\right)$ denoting the displacement amplitude of the longitudinal wave at the receiver $x_{j}$ in the frequency domain. 


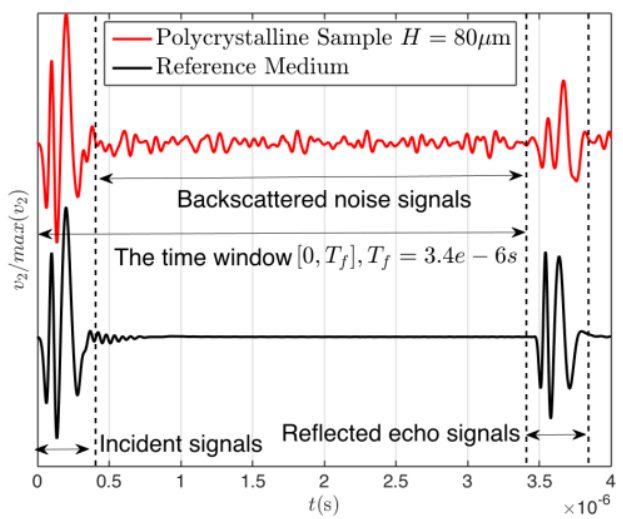

Fig. 12. Normalized velocity in $\boldsymbol{e}_{2}$ direction recorded at $10^{\text {th }}$ receiver in both polycrystalline (upper curve) and reference media (lower curve)

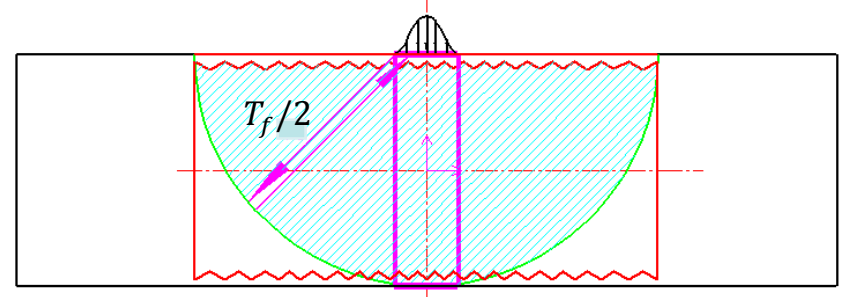

Fig. 13. Minimum and maximum limits $S_{p c M i n}$ and $S_{p c M a x}$ of polycrystalline surface involved by backscattering during the analysis period $\left[0, T_{f}\right]$

\section{$4 \quad$ Numerical evaluation of grain size effects on the backscattering}

In this section, numerical results of investigating grain size effects on the backscattering are presented. As indicated in Section 3.1, three grain sizes of $80 \mu \mathrm{m}, 160 \mu \mathrm{m}$ and $320 \mu \mathrm{m}$ are studied, ten random distributions of crystallographic orientations are calculated for each grain size. Different kinds of grain morphologies, such as regular and irregular hexagonal grain shapes as well as a random grain morphology generated by the Voronoi tessellation, are considered.

\subsection{Grain size effect}

The numerically and theoretically evaluated backscattering coefficients are firstly considered in the frequency domain (Section 4.1.1). Then the backscattered noise levels are measured and analyzed in the time domain (Section 4.1.2).

\subsubsection{Backscattering coefficient in the frequency domain}

Comparisons between theoretical and numerical estimates of the backscattering coefficient in the frequency domain for all the three grain sizes are shown in Fig. 14.(a)-(c). For the numerical results, three curves of respectively maximum values, mean values and minimum values of the backscattering coefficient for the ten random distributions of crystallographic orientations are plotted respectively in 
dash-dotted, solid and dotted lines. Circle markers are used on the curves of the mean values. The analytical predictions $\eta S_{p c M i n}$ and $\eta S_{p c M a x}$ are plotted in solid and dash lines.

For all the three considered grain sizes, the averaged response over ten samples obtained by the numerical simulations fluctuates between the two analytical bounds $\eta S_{p c M i n}$ and $\eta S_{p c M a x}$, its variation tendency with respect to the frequency is consistent with the analytical prediction. The backscattered energy increases with frequency whatever the grain size. However, in low frequency region, the larger the grain size is, the larger the backscattered noise levels are, while in high frequency region, the larger the grain size is, the less important the backscattered noise levels are. Moreover in high frequency region, the backscattering coefficient in the sample with the largest grains, i.e. $320 \mu \mathrm{m}$, becomes nearly constant while the backscattering coefficient of the other two samples with smaller grain sizes keeps increasing with frequency.

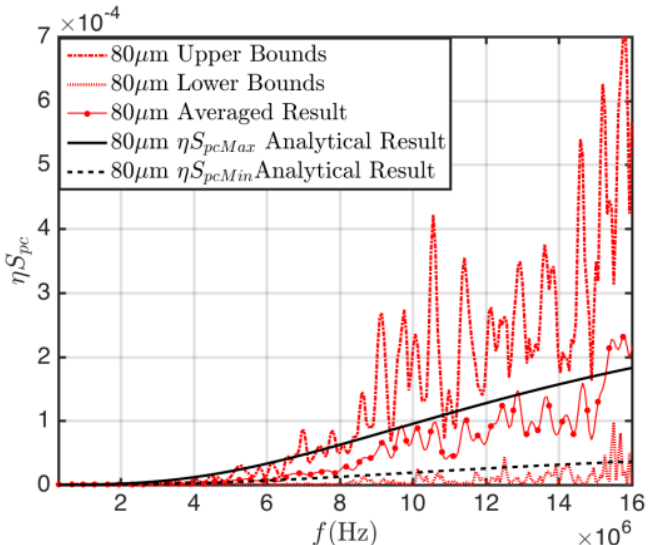

(a)

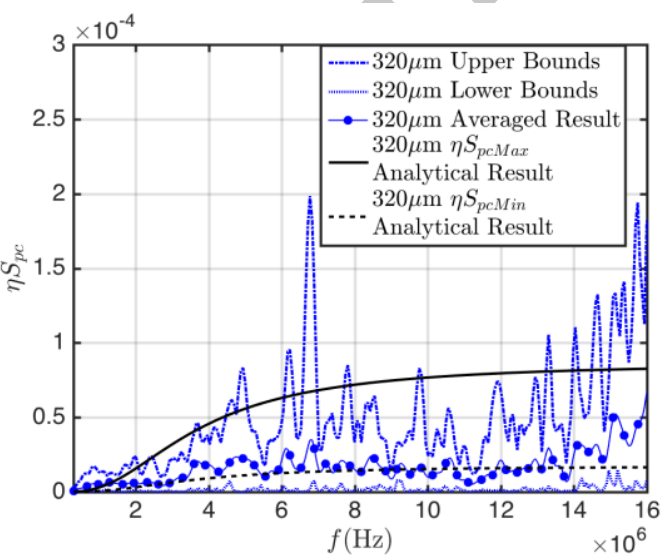

(b)
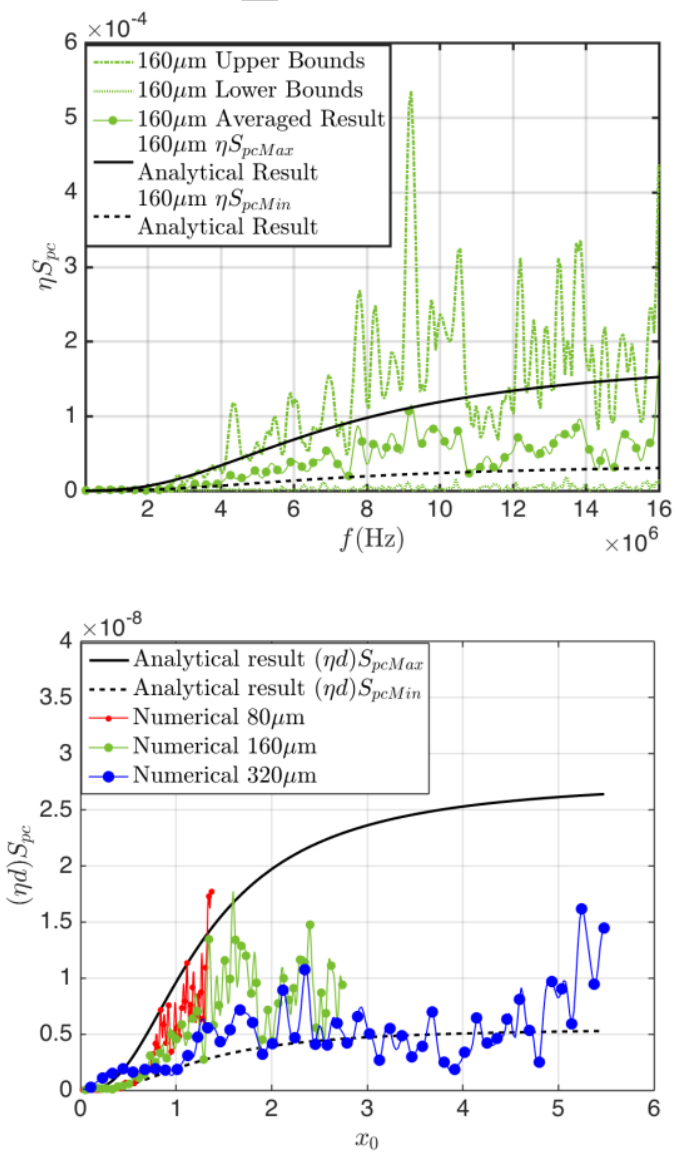

Fig. 14. Comparisons in the frequency domain between theoretical and numerical estimates. Backscattering coefficient for respectively three grain sizes (a) $80 \mu \mathrm{m}$, (b) $160 \mu \mathrm{m}$ and (c) $320 \mu \mathrm{m}$; (d) Backscattering coefficient per crystallite as a function of the normalized frequency $x_{0}$

Significant gaps between the curves of maximum values and minimum values of the backscattering coefficient for the ten studied samples are obtained and highlight important dispersions in backscattered noise levels between two random distributions of crystallographic orientations. More particularly, we remark that the maximum values of backscattering coefficient obtained by ten 
numerical samples are larger than the theoretical upper bound $\eta S_{p c M a x}$ in the case of grain sizes equal to $80 \mu \mathrm{m}$ and $160 \mu \mathrm{m}$ in high frequency range. This point will be further discussed in Section 4.2.

Otherwise, Fig. 14.(d) compares the master curves obtained theoretically and numerically, i.e. the previously compared curves are plotted again in terms of the backscattering coefficient per crystallite $(\eta d) S_{p c}$ and the normalized frequency $x_{0}=2 \pi d / \lambda_{0}$. The two master curves numerically calculated of grain sizes $80 \mu \mathrm{m}$ and $160 \mu \mathrm{m}$ are well superposed in the low frequency range $\left(x_{0}<0.4\right)$ and are consistent with the lower bound of theoretical prediction $(\eta d) S_{p c \text { Min }}$. Then they rapidly increase with slight fluctuation, keep being more or less superposed and are bounded by the lower and upper theoretical bounds. Finally in the high frequency range $\left(x_{0}>2\right)$ the two numerically calculated master curves of grain sizes of $160 \mu \mathrm{m}$ and $320 \mu \mathrm{m}$ gradually decrease and tend to the constant level defined by the lower theoretical bound $(\eta d) S_{p c \text { Min }}$ rather than the upper bound $(\eta d) S_{p c \text { Max }}$. This phenomenon has not been well understood yet. However we believe that the relatively high degree of anisotropy of the studied material may be the cause as the use of the Born approximation should be no more relevant [6].

\subsubsection{Backscattered noise levels in the time domain}

It is also interesting to measure the backscattered noise levels in the time domain. For a given frequency $f_{0}$, the root-mean-square (rms) positional average of the difference between the observed noise signal and the positional averaged noise level, i.e. $n_{r m s}\left(f_{0} ; t\right)$ is measured [31]:

$$
n_{r m s}\left(f_{0} ; t\right)=\left(\frac{1}{10 \times M} \sum_{j=1}^{10 \times M}\left(v_{2}^{B}\left(\boldsymbol{x}_{j}, f_{0} ; t\right)-b\left(f_{0} ; t\right)\right)^{2}\right)^{1 / 2}
$$

with

$$
b\left(f_{0} ; t\right)=\frac{1}{10 \times M} \sum_{j=1}^{10 \times M} v_{2}^{B}\left(\boldsymbol{x}_{j}, f_{0} ; t\right)
$$

where $10 \times M$ means the average is taken over all receivers and over all the ten samples, so we have in fact 220 measures. $v_{2}^{B}\left(\boldsymbol{x}_{j}, f_{0} ; t\right)$ is calculated by applying a frequency filtering, corresponding to the frequency $f$, to the velocity component $v_{2}^{B}\left(\boldsymbol{x}_{j} ; t\right)$ in $\boldsymbol{e}_{2}$ direction of the noise signal recorded at the jth receiver $\boldsymbol{x}_{j}$ [32]. $b\left(f_{0} ; t\right)$ is interpreted as the mean of the noise level at the time $t$ for a given frequency $f_{0}$ and would be zero if the total number of measures, equal to $10 \times M$ in the present work, is sufficiently large [31]. In this case, $n_{r m s}\left(f_{0} ; t\right)$ is identical to the standard deviation of the noise level. To eliminate the dependence of the noise level on the incident power, normalized rms noise level $N_{r m s}\left(f_{0} ; t\right)$ is defined [31]:

$$
N_{r m s}\left(f_{0} ; t\right)=n_{r m s}\left(f_{0} ; t\right) / E_{\max }\left(f_{0}\right)
$$

where $E_{\max }$ is taken equal to one half of the peak-to-peak amplitude of the incident signals for the frequency $f_{0}$. 
Fig. 15. presents, for the three grain sizes, the normalized rms noise level in the time domain for the two central frequencies $5 \mathrm{MHz}$ and $10 \mathrm{MHz}$ defined by the two incident Ricker signals. With the properties of the equivalent homogeneous medium and the emitter line width of the pressure loading, a limit of the Fresnel zone indicating the beginning of the far-field region is estimated theoretically and verified numerically to be between $0.6 \mu$ s and $1.1 \mu$ s. Therefore, the peaks of the curves in Fig. 15 are consistent with this limit. Afterwards, the drop with depth of noise levels is observed for two reasons: one associated with the attenuation and the other resulting from the beam spread. It is well known that the single Gaussian beam model describes the beam spread as inversely proportional to the frequency [1]. As the drop with depth at its beginning in the cases of grain sizes of $80 \mu \mathrm{m}$ and $160 \mu \mathrm{m}$ is more rapid for $f_{0}=10 \mathrm{MHz}$ than for $f_{0}=5 \mathrm{MHz}$ (Fig. 15), it seems that the attenuation would play a more important role for $f_{0}=10 \mathrm{MHz}$ than the beam spread. However this conclusion should be confirmed by further quantitative analyzes.

The highest noise level is given by the grain size of $160 \mu \mathrm{m}$ for $f_{0}=5 \mathrm{MHz}$ and by the grain size of $80 \mu \mathrm{m}$ for $f_{0}=10 \mathrm{MHz}$. By comparison with the theoretical results (Fig. 3.), it seems that the transition from the stochastic region to the geometric scattering regime should numerically occur earlier than the theoretical prediction. Indeed in Fig. 3. the theoretical curve for the grain size of $160 \mu \mathrm{m}$ is still above the one for the grain size of $80 \mu \mathrm{m}$, while in Fig. 15.(b)) the numerical simulation gives a noise level for the grain size of $160 \mu \mathrm{m}$ lower than the one for the grain size of $80 \mu \mathrm{m}$. For $f_{0}=10 \mathrm{MHz}$, the grain size of $320 \mu \mathrm{m}$ gives the lowest noise level, which is also in good agreement with the previous analysis in the frequency domain (Fig. 14.).

(a)

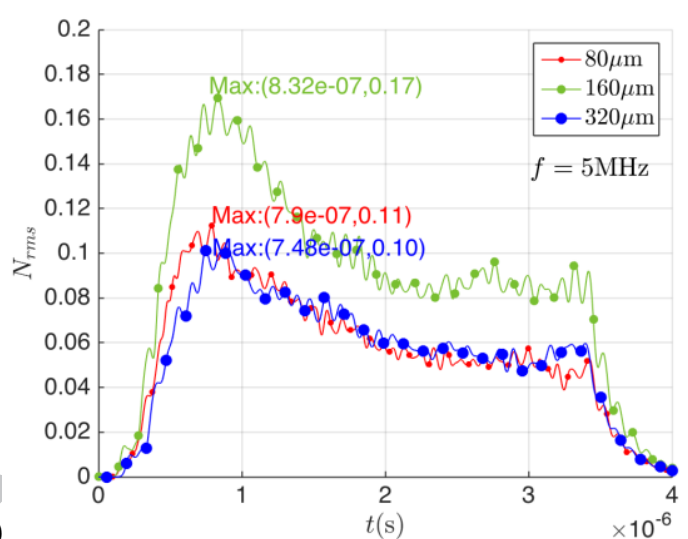

(b)

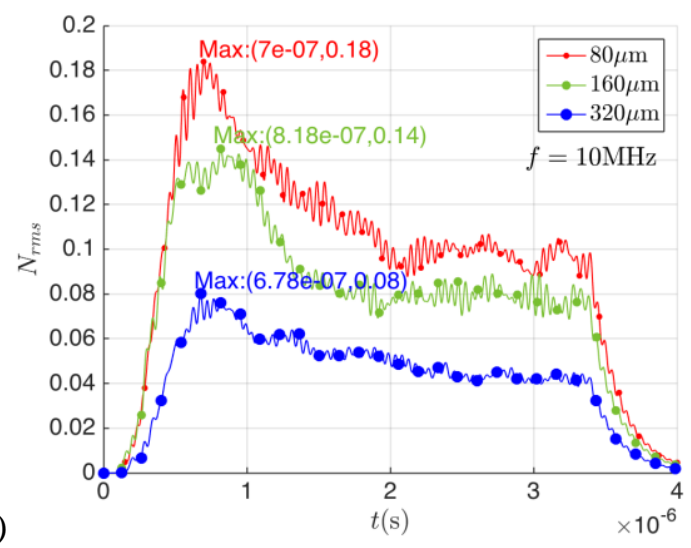

Fig. 15. Normalized rms noise level in the time domain for three grain sizes at the central frequencies (a) $f_{0}=5 \mathrm{MHz}$ and (b) $f_{0}=10 \mathrm{MHz}$.

The main purpose of this paper is the development of a numerical approach for estimating the diffusion coefficient in the frequency domain. Nevertheless the evolution in the time domain of grain noise levels is also very important and many theoretical, numerical and experimental works have been carried in the literature (e.g. [20, 31, 33-35]), further comparisons with the analytical models are to be 
done and constitute an interesting way to validate numerical tools. The numerical simulation would allow investigating in return the hypothesis used by the theoretical models.

\subsection{Grain boundary orientation effect}

To check possible numerical artifacts due to the shape of regular hexagonal grains, FE simulations are performed for the microstructures with irregular hexagonal grains and the Voronoitype random grain morphology presented in Section 3.1. Comparisons of the backscattering coefficient between these two microstructures and the one with single-size regular hexagonal grains for the grain size of $\langle H\rangle=80 \mu \mathrm{m}$ are shown in Fig. 16.(a). For the grain size of $\langle H\rangle=160 \mu \mathrm{m}$, only comparisons between regular and irregular hexagonal grain morphologies are considered in Fig. 16.(b). Good agreement is obtained with only slight deviations in the high frequency region of $f>10 \mathrm{MHz}$.

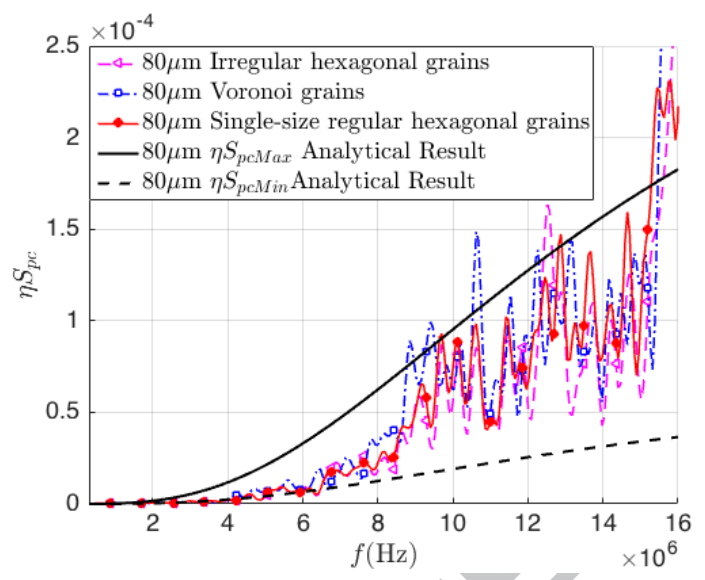

(b)

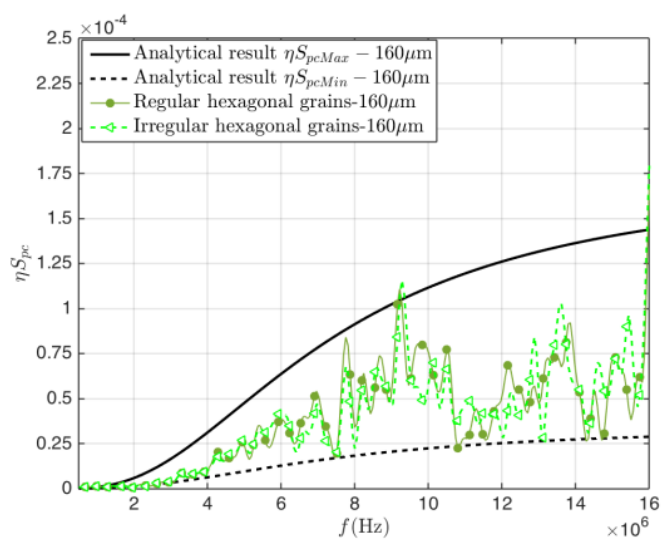

Fig. 16. Comparison of the backscattering coefficient between microstructures with (a) three different grain morphologies of averaged grain size of $80 \mu \mathrm{m}$ and (b) two different grain morphologies of averaged grain size of $160 \mu \mathrm{m}$.

The very small differences of the backscattering coefficient observed between the three different grain morphologies can be explained by considering the spatial correlation function. Indeed, according to (35) or (36), differences in the backscattering coefficient between microstructures containing different grain morphologies but with the same randomly-distributed crystallographic orientations are only due to the spatial correlation functions $W(\boldsymbol{r})=\sum_{I}\left\langle\gamma^{I}(\boldsymbol{x}) \gamma^{I}\left(\boldsymbol{x}^{\prime}\right)\right\rangle$, which depends upon the grain size and shape. Under the assumption that the media are statistically isotropic and ergodic, $W(\boldsymbol{r})$ has a spherical symmetry and depends only on $r=|\boldsymbol{r}|$ the distance between two points. Therefore it is convenient to calculate $W(r)$ in the wave propagation direction, which is the direction $\boldsymbol{e}_{2}$ in our study. As referred to Stanke [36], an approximation of $W(r)$ can be deduced considering a microstructure composed of a sufficiently large number $N$ of grains in the following way:

$$
W_{N}(r)=\left\langle\frac{\sum_{I=1}^{N}\left(c_{I}-r\right)^{+}}{\sum_{I=1}^{N} c_{I}}\right\rangle
$$


Here $c_{I}$ is the length of a line segment parallel to the propagation direction passing through one grain with its end points falling on the grain boundary, named chord length (Fig. 17.), $(\cdot)^{+}$is the positive part of $(\cdot)$ and $\langle\cdot\rangle$ represents the spatial average over the whole sample.

For the microstructure with single-size regular hexagonal grains, $W_{N}(r)$ is independent on $N$ and is therefore simple to calculate. For geometrically varying grains, it is possible to obtain the chord lengths in each grain along any line parallel to $\boldsymbol{e}_{2}$ but the computation amount is huge since a sufficiently large number of grains have to be processed. Thus, an alternative approach is proposed by considering an equivalent diameter $H_{I}$ of inscribed circle to the regular hexagonal grain with an identical surface area for the grain $g^{I}$ :

$$
W_{N}(r) \approx\left\langle\frac{\sum_{I=I}^{N}\left(H_{I}-r\right)^{+}}{\sum_{I=1}^{N} H_{I}}\right\rangle
$$

Indeed, under the assumption that the media are statistically isotropic and ergodic, (63) gives a good estimation of (64).

Spatial correlation functions for the microstructures with three different grain morphologies are presented in Fig. 18.. They are compared with the spatial correlation function of polycrystals containing random-shaped equiaxed grains with Poisson grain size distribution expressed in an inverse exponential form (39). It is obvious that the differences in $W(r)$ between the three microstructures with the same effective grain size are very small. Hence this study provides an analytical insight into little differences observed in the backscattering coefficient between them. Moreover, it is noticed that the differences between the regular hexagonal single-size grains and the theoretical model of randomly shaped and equiaxed grains with Poisson distribution are no more than $10 \%$. The maximum difference occurs at the distance equal to the mean grain size $80 \mu \mathrm{m}$.
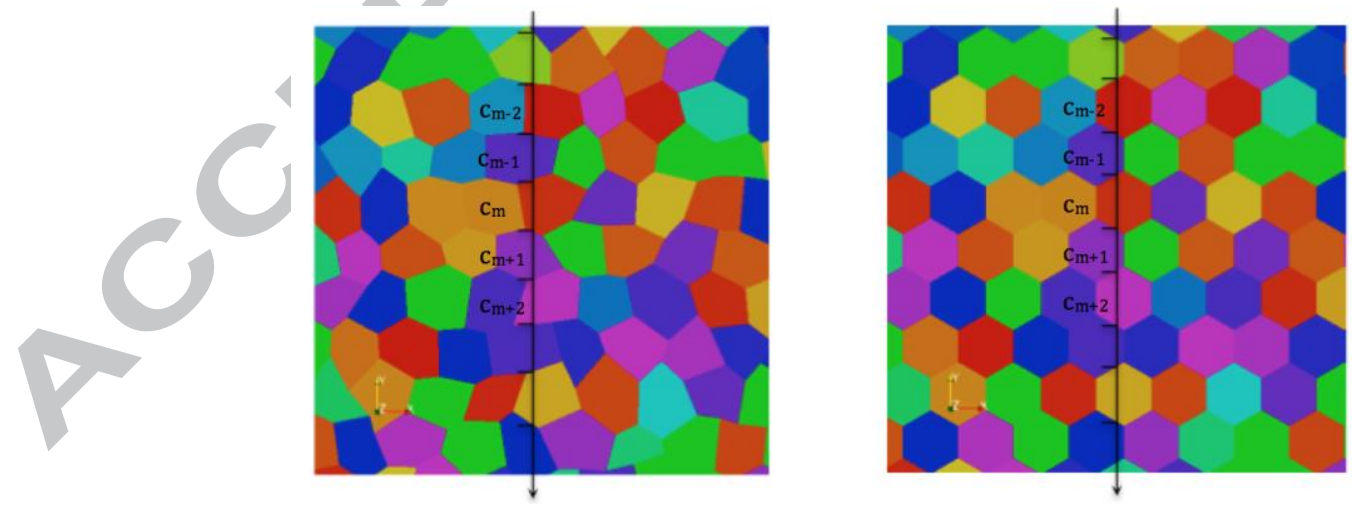

Fig. 17. Diagram of spatial correlation function measurement for microstructures with irregular or regular hexagonal grains 


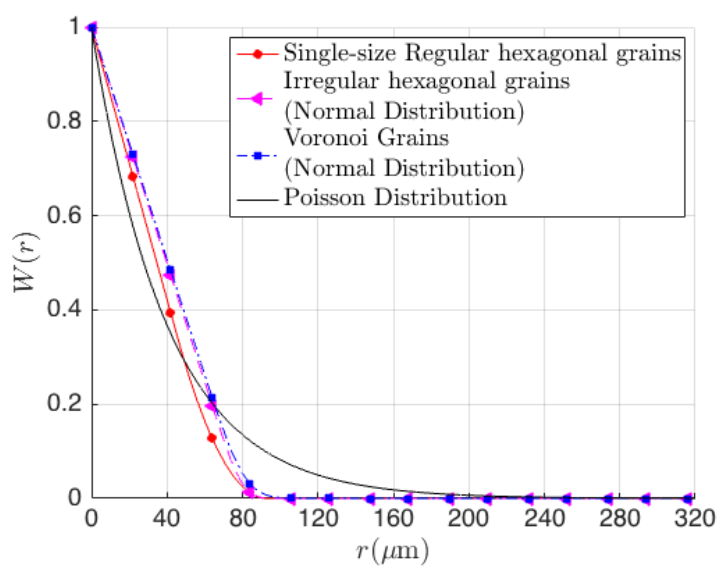

Fig.18. Comparison of the spatial correlation function between microstructures with four different grain morphologies: regular and irregular hexagonal grain morphologies, the Voronoi-type random grain morphologies with normal and Poisson grain size distribution.

Therefore it can be concluded that no numerical artifact results from the idealized microstructure composed of single-sized regular hexagonal grains. The large oscillations of the backscattering coefficient are then not due to any periodic morphology of the grain microstructure. The observed peaks and troughs of the backscattering, with a similar intensity whatever the grain shape, have probably a "physical" origin, which is discussed in the following section.

\subsection{Crystallographic misorientation effect}

The theoretical formulas give an averaged evaluation of the backscattering coefficient over all untextured distributions of crystallographic orientations and under the assumption of independent scattering approximation. Any microstructure should however deviate more or less from this averaged prediction. Fig. 19. presents, in the case of the grain size of $80 \mu \mathrm{m}$, large variations between the numerically calculated backscattering coefficient for samples having different distributions of crystallographic orientations. It is worth mentioning that all the different distributions give a nearly same Voigt average of elastic stiffness tensor. However the backscattering coefficient in the frequency domain for each sample fluctuates greatly up and down with erratically placed peaks and at some frequencies the backscattering coefficient can be very low and approach zero (Fig. 19.(a)). Numerical evaluations of the backscattering coefficient for one sample, then by averaging over five and ten samples are compared in Fig. 19.(b). As expected, increasing the number of samples can obviously smooth out fluctuations and improve the comparison between theoretical and numerical evaluations.

To provide some insight into the fluctuations of the backscattering coefficient with frequency, we consider in the following a group of seven adjacent grains of $80 \mu \mathrm{m}$, denoted $\Xi$ and embedded in the homogeneous reference medium (Fig. 20.(a)). Each grain has a randomly defined crystallographic orientation $\theta$ and a different level of phase velocity deviation $\Delta v_{q L}(\theta)=\left(v_{q L}(\theta)-v_{0 L}\right) / v_{0 L}$ from the reference medium along the $\boldsymbol{e}_{2}$ direction for the longitudinal wave. Actually, for a randomlyoriented polycrystalline titanium, $\Delta v_{q L}(\theta)$ has an upper bound of $+5.6 \%$ and a lower bound of $-5.2 \%$. 
For the grain whose centroid locates at the origin of the coordinate system, the phase velocity is chosen to approach to the one in the equivalent homogeneous medium, then the six adjacent grains are chosen to have two levels of phase velocity deviations approaching to the material limits, two negative levels of phase velocity deviations and two slight magnitude of phase velocity deviations. The seven grains are numbered as indicated in Fig. 20.(a) and their phase velocity deviation $\Delta v_{q L}(\theta)$ are respectively equal to $-0.06 \%,+5.3 \%,-4.1 \%,-0.8 \%,+0.5 \%,+1.7 \%,+4.5 \%$. According to the theoretical principle of the isolated scatterer model, eight numerical simulations are performed: One simulation with all seven grains involved and seven simulations each of which has only one grain involved without changing its location.

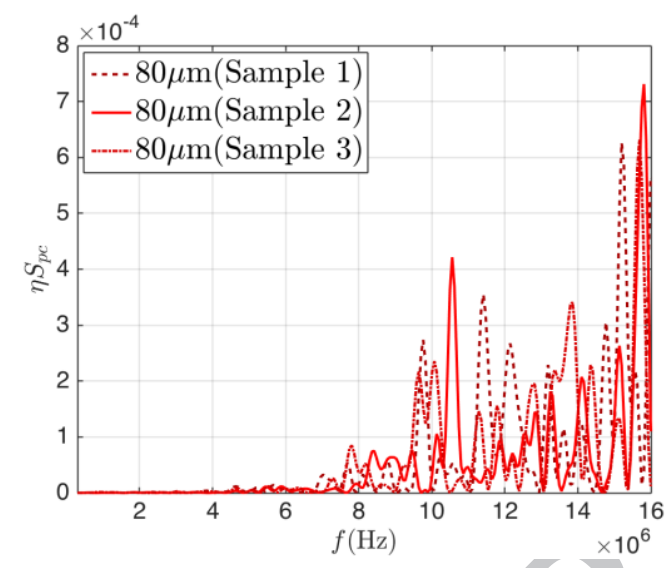

(a)
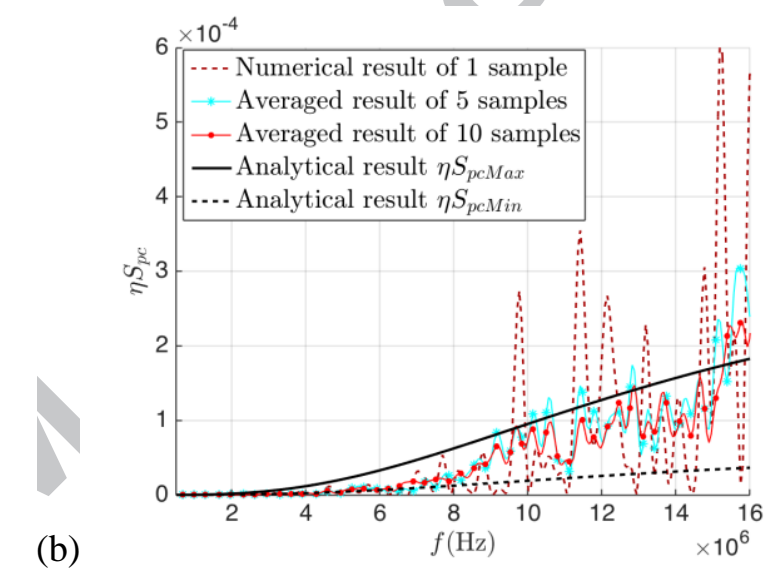

Fig. 19. Backscattering coefficient in the frequency domain for the grain size of $80 \mu \mathrm{m}$ : (a) Variations between three samples; (b) Effects of the number of samples to analyze on the averaged backscattering coefficient.

To illustrate the numerical results, Fig. 21. presents the time domain response recorded at the 10th receiver and the backscattering coefficient in the frequency domain for three grains: 2th, 3th and 6th. In the time domain, two primary noise signals scattered by a single grain are observed, which correspond respectively to longitudinal-to-longitudinal scattering and longitudinal-to-shear wave scattering. In the frequency domain, the backscattering coefficient remains oscillating but with very small amplitudes and is increasing with frequency on the whole. There's no more backscattering level fluctuating down to zero. It seems that a larger velocity deviation $\left|\Delta v_{q L}(\theta)\right|$ results in higher levels of backscattered noise signals in both time and frequency domains. Moreover with equal level of velocity deviation, a grain slower than the reference background seems to send higher level of noises backwards than a grain faster than the reference background.

Based on the independent scattering approximation using the isolated scatterer model, individual contributions of each grain to the backscattered noise signals are added independently $\sum_{I=1}^{7} u_{2}^{b}\left(\boldsymbol{x}_{10}, t, g^{I}\right)$. Fig. 21.(a) compares this sum to $u_{2}^{b}\left(\boldsymbol{x}_{10}, t, \Xi\right)$, which is the backscattered displacement fields in the $\boldsymbol{e}_{2}$ direction recorded at the $10^{\text {th }}$ receiver $\boldsymbol{x}_{10}$ for the medium $\Xi$, with all 
seven grains involved. Insignificant differences between them are observed, which are in fact the part due to the multiple scattering.

Otherwise in the frequency domain, the backscattering coefficient directly calculated using the numerical simulation involving all the seven grains as $\eta(\omega, \Xi)=\frac{\beta}{V_{p c}} \delta S(\omega, \Xi) \delta S^{*}(\omega, \Xi)$, can be compared to the average over independent contribution of each grain $\frac{\beta}{V_{g}}\left\langle\delta S\left(\omega, g^{I}\right) \delta S^{*}\left(\omega, g^{I}\right)\right\rangle$, which is exactly the application of the independent scattering approximation. Significant differences are then observed between them and also with the analytical prediction (Fig. 21.(b)).

To explain those differences, the following estimation of $\delta S(\omega, \Xi) \delta S^{*}(\omega, \Xi)$ in the frequency domain is written based on the assumption that the scattered noise signal $\boldsymbol{u}^{b}\left(\boldsymbol{x}_{j}, t, \Xi\right)$ recorded at $j^{\text {th }}$ receiver is the sum of the contribution of each individual grain $\boldsymbol{u}^{b}\left(\boldsymbol{x}_{j}, t, g^{I}\right)$ :

(a)

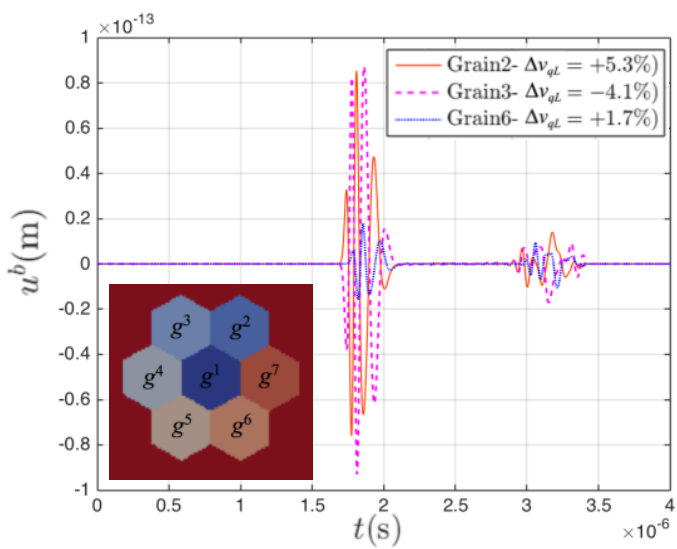

(b)

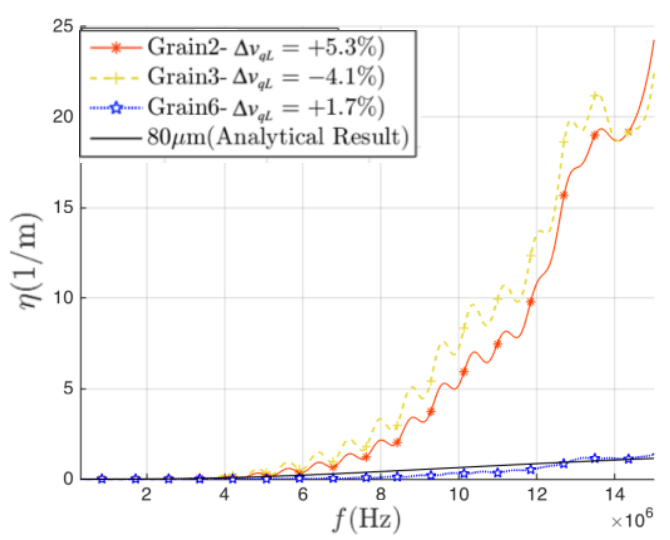

Fig. 20. (a) Time-series scattered noise signals at the $10^{\text {th }}$ receiver and (b) frequency dependence of the backscattering coefficient for three isolated grains within a homogeneous isotropic medium

(a)

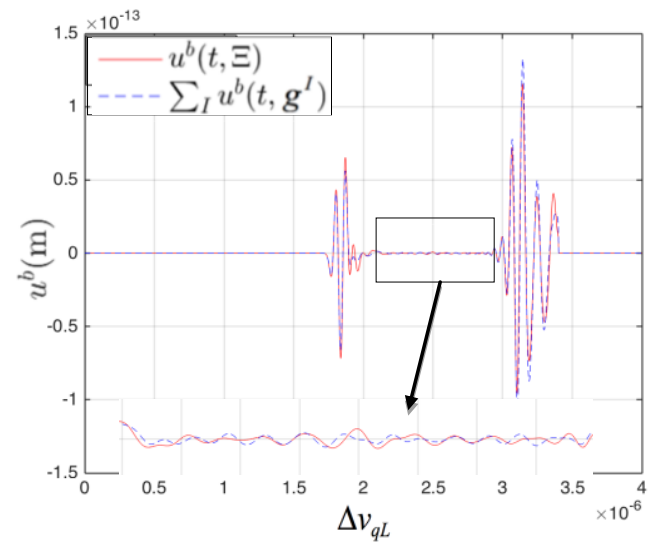

(b)

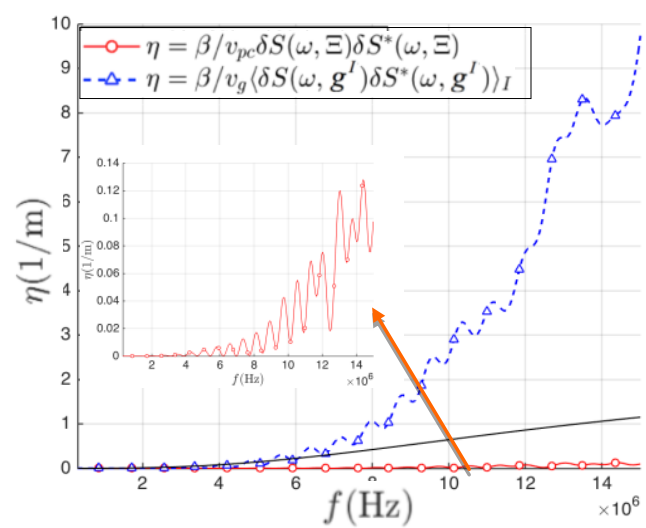

Fig. 21. Analysis of the independent scattering approximation. (a) Comparison of time domain signals recorded at the $10^{\text {th }}$ receiver $\boldsymbol{x}_{10}$ between $u_{2}^{b}\left(\boldsymbol{x}_{10}, t, \Xi\right)$ and $\sum_{I=1}^{7} u_{2}^{b}\left(\boldsymbol{x}_{10}, t, g^{I}\right)$; (b) Comparison of backscattering coefficient calculated using $\delta S(\omega, \Xi) \delta S^{*}(\omega, \Xi) / V_{\Xi}$ and $\left\langle\delta S\left(\omega, g^{I}\right) \delta S^{*}\left(\omega, g^{I}\right)\right\rangle / V_{g}$

$$
\delta S(\omega, \Xi) \delta S^{*}(\omega, \Xi)=\left(\sum_{i} \boldsymbol{T}\left(\boldsymbol{x}_{i}, \omega\right) \cdot\left(\sum_{I} \boldsymbol{u}^{b}\left(\boldsymbol{x}_{i}, \omega, g^{I}\right)\right)\right)\left(\sum_{j} \boldsymbol{T}\left(\boldsymbol{x}_{j}, \omega\right) \cdot\left(\sum_{J} \boldsymbol{u}^{b}\left(\boldsymbol{x}_{j}, \omega, g^{J}\right)\right)\right)^{*}(\Delta x)^{2}
$$




$$
\begin{aligned}
& =\sum_{I=J}\left(\sum_{i} \boldsymbol{T}\left(\boldsymbol{x}_{i}, \omega\right) \cdot \boldsymbol{u}^{b}\left(\boldsymbol{x}_{i}, \omega, g^{I}\right)\right)\left(\sum_{j} \boldsymbol{T}\left(\boldsymbol{x}_{j}, \omega\right) \cdot \boldsymbol{u}^{b}\left(\boldsymbol{x}_{j}, \omega, g^{J}\right)\right)^{*}(\Delta x)^{2} \\
& +\sum_{I \neq J}\left(\sum_{i} \boldsymbol{T}\left(\boldsymbol{x}_{i}, \omega\right) \cdot \boldsymbol{u}^{b}\left(\boldsymbol{x}_{i}, \omega, g^{I}\right)\right)\left(\sum_{j} \boldsymbol{T}\left(\boldsymbol{x}_{j}, \omega\right) \cdot \boldsymbol{u}^{b}\left(\boldsymbol{x}_{j}, \omega, g^{J}\right)\right)^{*}(\Delta x)^{2} \\
& =\frac{V_{\Xi}}{V_{g}}\left\langle\delta S\left(\omega, g^{I}\right) \delta S^{*}\left(\omega, g^{I}\right)\right\rangle+\sum_{I \neq J} \delta S\left(\omega, g^{I}\right) \delta S^{*}\left(\omega, g^{J}\right)
\end{aligned}
$$

where $\Delta x$ is the distance between two adjacent receivers. We note that (64) is true because the stress vector $\boldsymbol{T}\left(\boldsymbol{x}_{i}, \omega\right)=\boldsymbol{T}^{0}\left(\boldsymbol{x}_{i}, \omega\right)$ is prescribed on the emitter line and is therefore identical in all the simulations (see (49)). Two terms are therefore emphasized in (64), an auto-correlation term of each grain and a cross-correlation term between different grains. When the number of grains contributing to noise echo signals tends to infinity, the cross-correlation term tends to zero, according to the assumption that $\left\langle\delta \boldsymbol{C}^{I} \otimes \delta \boldsymbol{C}^{J}\right\rangle=0$ when $I \neq J$. Thereby the incoherent sum of grain contribution $\left\langle\delta S\left(\omega, g^{I}\right) \delta S^{*}\left(\omega, g^{I}\right)\right\rangle / V_{g}$ should be a good approximation to $\delta S(\omega, \Xi) \delta S^{*}(\omega, \Xi) / V_{\Xi}$, which is far from the case with only seven grains. In the same way, only in the case of a sufficiently large number of grains contributing to the received noise echo signals, the backscattering coefficient estimated by using $\left\langle\delta S\left(\omega, g^{I}\right) \delta S^{*}\left(\omega, g^{I}\right)\right\rangle / V_{g}$ would be comparable to the analytical prediction. Otherwise the cross-correlation term leads to oscillations due to the alternatively positive and negative phase velocity deviations and results in fluctuations in the backscattering coefficient for a sample, as the volume of grains travelled by the ultrasonic beam always contains only a limited number of grains.

We believe that the number of grains contributing to the backscattering has a significant influence on the oscillatory levels of the numerically estimated backscattering coefficient. Increasing the number of studied samples should lead to a smoother curve of the backscattering coefficient. Generally Margetan et al. propose to use 100 to 1000 waveforms per analysis, given that the receiver positions are chosen to be as widely spaced as possible to reduce the interactions [31]. We note that for the averaged numerical results shown in Fig.14, with 22 receivers and 10 samples calculated, 220 waveforms have been analyzed.

To investigate how many realizations need to be executed to get an accurate prediction in terms of grain noise levels of an ensemble of various microstructures, ten more realizations of the microstructure with the grain size of $80 \mu \mathrm{m}$ are carried out, with 22 noise waveforms for each realization acquired. Convergence at different frequencies of the backscattering coefficient as a function of the number of waveforms is analyzed (Fig. 22). It is noticeable that the lower the frequency, the more rapidly the grain noise measurements converge. Specifically, for the considered titanium alloy, there is a beginning of convergence with about 100 noise waveforms for the frequency range $f<6 \mathrm{MHz}\left(x_{0}<0.5\right)$, about 250 noise waveforms for the frequency range $f<12 \mathrm{MHz}\left(x_{0}<\right.$ 1.0). Obviously, more waveforms are needed for higher frequencies. In summary, the convergence between ten and twenty realizations is obvious, however, future studies are necessary to know precisely the number of realizations needed to achieve convergence for a required accuracy and for a frequency range of interest. Otherwise, it is important to emphasize that in a real polycrystal, the 
number of grains in the investigated volume is limited and so FE simulation on a limited volume could be a better way to understand and estimate variabilities between the experimental results than the analytical approach assuming an infinite medium.

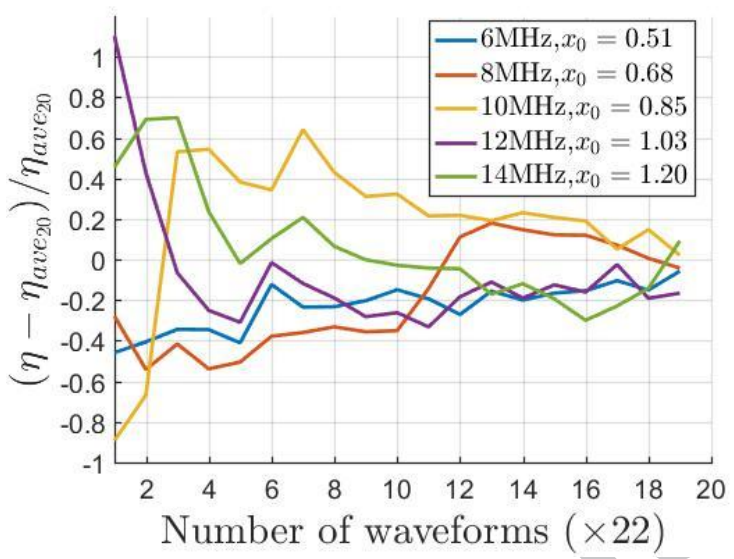

Fig. 22 Convergence of the numerical evaluation of the backscattering coefficient of an ensemble of microstructures with the grain size of $80 \mu \mathrm{m}$ in terms of the number of analyzed waveforms

\section{Conclusions}

A versatile approach to numerically evaluate the scattering coefficient in polycrystalline materials has been proposed. It is based on the reciprocity theorem and on the full-field FE modeling in the time domain of the ultrasonic wave propagation in polycrystalline materials. It is applicable for any polycrystal regardless of its anisotropy, crystallographic or morphological textures (grain size, grain elongation, etc.). The effects of multiple scattering is automatically included in the measure of the scattering coefficient as actual scattered fields are solved by the FE simulation, which makes it a powerful and promising tool to investigate real polycrystalline materials even with strong-scattering microstructures. We note the developed numerical approach is capable to simulate more complex microstructures. For example, microstructures with bimodal grain-size distributions have been studied [28]. Numerical tools have also been developed to consider real microstructures defined by EBSD measurements.

In the $2 \mathrm{D}$ case of single-phase, un-textured and equiaxed polycrystals, the backscattering has been analyzed analytically and numerically in a unified theoretical framework. Coherent variation tendency of the backscattering coefficient in the frequency domain was observed between the numerical measurements and the analytical prediction. Effects of the grain size, the grain boundary orientations and the distribution of crystallographic orientations in the backscattering have been numerically investigated. Discussions on the two-point spatial correlation functions of microstructures have been made to improve the understanding of slight influence of grain boundary orientations.

As indicated in Introduction, the analytical formulas for the diffusion coefficient developed within the same framework based on the reciprocity has been extended to general pitch-catch 
configurations [11], we believe that the proposed numerical evaluation approach would be versatile enough to consider these general configurations. However, mode-conversion scattering measurements are more complicated, so the numerical approach should be validated and perhaps need to be enriched. The numerical simulation that allows accessing full-field data should further progress the understanding of mode-conversion scattering phenomena and better quantify each conversion mechanism.

Finally, it is important to perform 3D numerical simulations, as polycrystalline textures are inherently a 3D concept. For example, recent research works have been developed by Van Pamel et al. $[15,37]$ to compare effects of the $2 \mathrm{D}$ and $3 \mathrm{D}$ modelings on the ultrasonic scattering. The $2 \mathrm{D}$ analytical formulas we developed for both diffusion and attenuation coefficients (see [2] for the 2D formulas of the attenuation coefficient) confirm the dimensionality of the ultrasonic scattering within polycrystalline materials. However, the 2D modeling is still interesting and useful. Indeed, it can be relatively pertinent in particular configurations, such as the case of polycrystals composed of very elongated grains and inspected with ultrasonic beams perpendicular to the longest axis of the grains. Otherwise, the 2D modeling allows efficient capture of some key features of ultrasonic scattering, such as the transition between different scattering regimes, which are similar and so representative for the 3D scattering phenomena.

\section{Acknowledgments}

The authors wish to thank the French National Centre for Scientific Research (CNRS), CentraleSupélec and the China Scholarship Council (CSC) for their financial supports of this research. They also acknowledge fruitful discussions with Dr. D. Solas mainly regarding a convenient choice for random distribution of crystallographic orientations.

\section{References}

[1] J. E. Gubernatis, E. Domany, J.A. Krumhansl. (1977). Formal aspects of the theory of the scattering of ultrasound by flaws in elastic materials. Journal of applied physics , 48 (7), 2804-2811.

[2]F. E. Stanke, G. S. Kino. (1984). A unified theory for elastic wave propagation in polycrystalline materials. The Journal of the Acoustical Society of America, 75 (3), 665-681.

[3]A. B. Bhatia. (1959). Scattering of high-frequency sound waves in polycrystalline materials. The Journal of the Acoustic Society of America, 31(1), 16-23.

[4] S. Hirsekorn. (1982). The scattering of ultrasonic waves by polycrystals. The Journal of the Acoustic Society of America, 72(3), 1021-1031.

[5]R. L. Weaver. (1990). Diffusivity of ultrasound in polycrystals. Journal of the Mechanics and Physics of Solids, 38(1), 55-86.

[6] J. E. Gubernatis, E. Domany, J. A. Krumhans, M. Huberman. (1977). The Born approximation in the theory of the scattering of elastic waves by flaws. Journal of Applied Physics , 48 (7), 2812-2819.

[7] Y. K. Han. (1997). Ultrasonic backscattering in duplex microstructures: theory and application to titanium alloys. Metallurgical and Materials Transactions A, 28 (1), 91-104.

[8]B. A. Auld. (1979). General electromechanical reciprocity relations applied to the calculation of elastic wave scattering coefficients. Wave motion , 1 (1), 3-10. 
[9] G. S. Kino. (1978). The application of reciprocity theory to scattering of acoustic waves by flaws. Journal of Applied Physics , 49 (6), 3190-3199.

[10] J. H. Rose. (1992). Ultrasonic backscatter from microstructure. Review of Progress in Quantitative Nondestructive Evaluation, 11B, pp. 1677-1684.

[11]F. J. Margetan, L. Yu, R. B. Thompson. (2005). Computation of Grain-Noise Scattering Coefficients for Ultrasonic Pitch/Catch Inspections of Metals. Review of Progress in Quantitative Nondestructive Evaluation. 760, pp. 1300-1307. AIP Publishing.

[12] G. Ghoshal, J. A. Turner, R. L. Weaver. (2009). Wigner distribution of a transducer beam pattern within a multiple scattering formalism for heterogeneous solids. Journal of the Acoustical Society of America, 122(4), 2009-2021.

[13] P. Hu, J. A. Turner. (2015). Contribution of double scattering in diffuse ultrasonic backscatter measurements. Journal of the Acoustical Society of America, 137(1), 321-334.

[14] B. Tie, D. Solas, J. Thébault, C. Rey, T. Baudin, A. Mouronval, Numerical modeling of ultrasonic wave propagation in polycrytalline materials, 10th French Congress of Acoustics (CFA2010), https://hal.archives-ouvertes.fr/hal-00550917 (in French)

[15] A. Van Pamel, C. R. Brett, P. Huthwaite, M. J. Lowe. (2015). Finite element modelling of elastic wave scattering within a polycrystalline material in two and three dimensions. The Journal of the Acoustical Society of America , 138 (4), 2326-2336.

[16] S. Shahjahan, F. Rupin, A. Aubry, B. Chassignole, T. Fouquet, T., A. Derode. (2014). Comparison between experimental and 2-D numerical studies of multiple scattering in Inconel. Ultrasonics , 54 (1), 358-367.

[17] J. Thébault. (2009). Experimental and numerical studies of the effects of heterogeneities of deformation and recrystallization on the mechanical properties of superalloys, PhD Thesis (In French), Ecole Centrale Paris.

[18] O. Dupond, N. Feuilly, B. Chassignole, T. Fouquet, J. Moysan, J., G. Corneloup. (2011). Relation between ultrasonic scattering and microstructure of polycrystalline materials. Journal of Physics: Conference Series. 269, p. 012010. IOP Publishing.

[19] G. Ghoshal, J. A. Turner. (2009). Numerical model of pressure wave scattering in polycrystals. Ultrasonics, Ferroelectrics, and Frequency Control, IEEE Transactions on , 56 (7), 1419-1428.

[20] G. Ghoshal, J. A. Turner. (2009). Diffuse ultrasonic backscatter in a two-dimensional domain. Acta Mechanica, 205(1), 35-49.

[21] Nicoletti, D., \& Anderson, A. (1997). Determination of grain-size distribution from ultrasonic attenuation: transformation and inversion. The Journal of the Acoustical Society of America, 101(2), 686-689.

[22] Garcin, T., Schmitt, J. H., and Militzer, M. (2016). In-situ laser ultrasonic grain size measurement in superalloy INCONEL 718. Journal of Alloys and Compounds, 670, 329-336.

[23] J.P. Monchalin (2004). Laser-ultrasonics: From the laboratory to industry. Rev. Quant. Nondestruct. Eval. 23, 3-31.

[24] Achenbach, J. D. (2003). Reciprocity in Elastodynamics. Cambridge University Press.

[25] A. C. Eringen, E. S. Suhubi, Elastodynamics, Vol. 2. Linear theory. (1975) New York: Academic Press.

[26] W. Petry, A. Heiming, J. Trampenau, M. Alba, C. Herzig, H. R. Schober, \& G. Vogl (1991). Phonon dispersion of the bcc phase of group-IV metals. I. bcc titanium. Physical Review B, 43(13), 10933.

[27] B. Tie, D. Aubry. (2006). Adaptive time discontinuous Galerkin method for numerical modeling of wave propagation in shell and 3D structures. European Journal of Computational Mechanics/Revue Européenne de Mécanique Numérique, 15(6), 729-757.

[28] X. Bai. (2017). Finite element modeling of ultrasonic wave propagation in polycrystalline materials. PhD thesis, CentraleSupélec, Université Paris-Saclay, France.

[29] C. M. Kube, J. A. Turner. (2015). Ultrasonic attenuation in polycrystals using a self-consistent approach. Wave Motion, 57, 182-193.

[30] J. L. Rose. Ultrasonic guided waves in solid media. (2014) New York: Cambridge University Press. 
[31]F. J. Margetan, R. B. Thompson, I. Yalda-Mooshabad. (1994). Backscattered microstructural noise in ultrasonic toneburst inspections. Journal of nondestructive evaluation, 13(3), 111-136.

[32] P. M. Beaufils, M. Rami. (1988). Numerical filtering (Le filtrage numérique), No. 04, QA276, B43 (in French).

[33] G. Ghoshal, J. A. Turner. (2010) Diffuse ultrasonic backscatter at normal incidence through a curved interface. The Journal of the Acoustical Society of America, 128(6), 3449-3458.

[34] G. Ghoshal, M. L. Oelze. (2012). Time domain attenuation estimation method from ultrasonic backscattered signals. The Journal of the Acoustical Society of America, 132(1), 533-543.

[35] X. B. Li, Y. F. Song, A. P. Arguelles, J. A. Turner. (2017). Diffuse ultrasonic backscatter using a multi-Gaussian beam model. The Journal of the Acoustical Society of America, 142(1), 195-205.

[36]F. E. Stanke. (1986). Spatial autocorrelation functions for calculations of effective propagation constants in polycrystalline materials. The Journal of the Acoustical Society of America, 80(5), 14791485.

[37] A. Van Pamel, P. B. Nagy, M. J. S. Lowe. (2016). On the dimensionality of elastic wave scattering within heterogeneous media. constants in polycrystalline materials. The Journal of the Acoustical Society of America , 140(6), 4360-4366. 
Article: Finite element modeling of grain size effects on the ultrasonic microstructural noise backscattering in polycrystalline materials

Authors: X. Bai, B. Tie ${ }^{l}$, J.-H. Schmitt, D. Aubry

\section{Highlights:}

- An original FE approach to quantify the ultrasonic noise scattering is proposed.

- The use of the reciprocity theorem guarantee the versatility of the approach.

- A frequency-dependent ratio is derived between the 2D and 3D noise measures.

- Grain size effects on backscattered noise levels in polycrystals are quantified.

- Grain structure effects (regular hexagons, Voronoï tessellation) are investigated.

${ }^{1}$ Corresponding author.

E-mail: bing.tie@centralesupelec.fr 